\title{
The Peano Software-Parallel, Automaton-based, Dynamically Adaptive Grid Traversals
}

\author{
TOBIAS WEINZIERL, Department of Computer Science, Durham University
}

\begin{abstract}
We discuss the design decisions, design alternatives, and rationale behind the third generation of Peano, a framework for dynamically adaptive Cartesian meshes derived from spacetrees. Peano ties the mesh traversal to the mesh storage and supports only one element-wise traversal order resulting from space-filling curves. The user is not free to choose a traversal order herself. The traversal can exploit regular grid subregions and shared memory as well as distributed memory systems with almost no modifications to a serial application code. We formalize the software design by means of two interacting automata-one automaton for the multiscale grid traversal and one for the application-specific algorithmic steps. This yields a callback-based programming paradigm. We further sketch the supported application types and the two data storage schemes realized before we detail high-performance computing aspects and lessons learned. Special emphasis is put on observations regarding the used programming idioms and algorithmic concepts. This transforms our report from a "one way to implement things" code description into a generic discussion and summary of some alternatives, rationale, and design decisions to be made for any tree-based adaptive mesh refinement software.
\end{abstract}

CCS Concepts: • Mathematics of computing $\rightarrow$ Solvers; $\bullet$ Computing methodologies $\rightarrow$ Multiscale systems; Shared memory algorithms; Massively parallel algorithms; Massively parallel and high-performance simulations;

Additional Key Words and Phrases: Software, spacetree, adaptive mesh refinement, parallel multiscale grid traversal

\section{ACM Reference format:}

Tobias Weinzierl. 2019. The Peano Software-Parallel, Automaton-based, Dynamically Adaptive Grid Traversals. ACM Trans. Math. Softw. 45, 2, Article 14 (April 2019), 41 pages.

https://doi.org/10.1145/3319797

\section{INTRODUCTION}

Dynamically adaptive grids are the mortar and catalyst of mesh-based scientific computing and thus important to a large range of scientific and engineering applications. They enable scientists

The author appreciates support received from the European Unions Horizon 2020 research and innovation programme under grant agreement No 671698 (ExaHyPE). This work made use of the facilities of the Hamilton HPC Service of Durham University. The author furthermore gratefully acknowledges the Gauss Centre for Supercomputing e.V. (www.gauss-centre. eu) for funding this project by providing computing time on the GCS Supercomputer SuperMUC at Leibniz Supercomputing Centre (www.lrz.de). Finally, this manuscript particularly has been benefitting from the support of the RSC Group who granted us early access to their KNL machines. All underlying software is open source (Weinzierl et al. 2015).

Authors' address: T. Weinzierl, Department of Computer Science, Durham University, DH1 3LE Durham, Great Britain; email: tobias.weinzierl@durham.ac.uk.

Permission to make digital or hard copies of all or part of this work for personal or classroom use is granted without fee provided that copies are not made or distributed for profit or commercial advantage and that copies bear this notice and the full citation on the first page. Copyrights for components of this work owned by others than ACM must be honored. Abstracting with credit is permitted. To copy otherwise, or republish, to post on servers or to redistribute to lists, requires prior specific permission and/or a fee. Request permissions from permissions@acm.org.

(c) 2019 Association for Computing Machinery.

0098-3500/2019/04-ART14 \$15.00

https://doi.org/10.1145/3319797 
and engineers to solve problems with high accuracy as they invest grid entities and computational effort where they pay off most. Naturally, these regions may change over time for time-dependent problems and may change throughout a solve process due to error estimators. The design of meshing software for dynamically adaptive grids is non-trivial, since it has to support a magnitude of advanced algorithmic building blocks such as discretization, refinement criteria, solver steps, or visualization. It has to facilitate functional diversity and be accessible. At the same time, mesh storage, administration, and processing have to meet efficiency and concurrency requirements of high-performance computing (HPC). One specific yet popular meshing paradigm that tries to meet these criteria and is the subject of study here is spacetrees. We use the term as generalization for quadtrees, octrees, and related data structures. They yield adaptive Cartesian grids (Burstedde et al. 2011; Gadeschi et al. 2015; Griebel and Zumbusch 1999; Khokhlov 1998; Lashuk et al. 2012; Robey et al. 2013; Sampath et al. 2008; Sundar et al. 2008; Tumblin et al. 2015; Weinzierl and Mehl 2011).

This article orbits around the spacetree software Peano (Weinzierl et al. 2015), which is available in its third generation. The first generation was a set of proof-of-concept codes tackling various challenges with different core code routines (e.g., Bungartz et al. (2006) and Mehl et al. (2006)). A second generation fused these concepts into one code base made primarily for CFD-type applications, although some generic software rationale have been reported ((Bungartz et al. 2011, 2010b; Weinzierl 2009; Weinzierl and Mehl 2011) and others). The third generation is a complete rewrite that focuses on an abstraction from any application, offers different memory organization schemes and emphasizes scalability. Our objective is twofold: On the one hand, we want to present this third generation of the software as well as the underlying rationale and, thus, sketch its potential. While Peano is used as workhorse for our own projects (Bader et al. 2015; Eckhardt and Weinzierl 2010; Reps and Weinzierl 2017; Schreiber et al. 2013a; Weinzierl and Weinzierl 2018; Weinzierl et al. 2014, 2016), making it freely available allows other groups to benefit from the development effort, too. For this, however, its design philosophy has to be described explicitly. Academics have to be enabled to assess whether it suits particular needs and whether certain design decisions make it inferior or superior to other codes. On the other hand, we want to identify and outline design and realization patterns (Gamma et al. 1994) for tree-based adaptive mesh refinement (AMR). Given the popularity of this meshing paradigm, our manuscript brings together and compares fundamental decisions to be made by any developer. Many of these comparisons and classifications have, to the best of our knowledge, not been done before in a concentrated effort.

While we work application generic, Peano does not strive for the flexibility and diversity along the lines of other solutions such as Adams et al. (2016b), Bangerth et al. (2016), Bastian et al. (2008a), Burstedde et al. (2011), de St. Germain et al. (2000), Feichtinger et al. (2011), Sampath et al. (2008), Teunissen and Ebert (2017) or other projects such as described and cited in Deiterding (2005), Dreher and Grauer (2005), and Dubey et al. (2016). This list is not comprehensive. Our approach ties the mesh traversal and programming model to the mesh structure: The user is not allowed to navigate through the grid freely. Instead, the grid traversal, i.e., the sequence in which grid entities are processes, is prescribed. This poses a restriction that may force algorithms to be redesigned, but it allows users to focus on which algorithmic steps are mapped onto the processing of which grid entity (vertex or cell, e.g.) and which temporal and data dependencies between these steps exist. How the processing is realized is hidden. This picks up recent trends in task-based computing and has successfully been used in various spacetree and non-spacetree codes such as Meister et al. (2012) and Weinbub et al. (2014). A minimalist set of constraints prescribing a partial order allows us to tune the grid traversal. It further enables us to hide concurrent execution from the application codes. It is one goal of this article to highlight how such a restrictive programming model interacts with other well-known concepts such as spacetree linearization based upon space-filling curves 
(Sundar et al. 2008), various discretization and data modeling choices, persistent data storage, domain decomposition, and task processing. Since the traversal invokes user-defined operations and disallows users to control the traversal, our programming paradigm can be summarizes by the Hollywood principle: Don't call us, we'll call you (Sweet 1985).

Many other implementation choices would have been possible, so a description of the chosen approaches and the motivation is of use to other spacetree code developers facing similar challenges. Our article contains a brief introduction of the grid data structure and the two grid enumeration schemes we rely on. The main part of the manuscript starts from a description of our callbackbased programming model (Section 3). This allows us to clarify which classes of applications are supported by the code as well as application limitations. In Section 4, we review some data storage paradigms for spacetrees and classify the two storage variants offered by Peano: stream- and heapbased persistency. Total linearization of the tree through space-filling curves (SFCs) enables us to encode the grid data and adjacency with minimal memory and to stream it through the processor. Holding a tree as stream rather than a pointer data structure is a popular technique and, thus, can be called a design pattern (Gamma et al. 1994). Our manuscript applies the well-known linearization pattern to multilevel grids and details the relation to depth-first (DFS) and breadth-first (BFS) searches. While depth-first linearization yields vertically integrated (Adams et al. 2016a) grid traversals-multiple levels are processed by one mesh sweep-it lacks concurrency for traditional stencil codes, and we thus derive a hybrid of DFS and BFS. Three sections are dedicated to a discussion of memory movement and administration minimization (Section 5) as well as the supported shared memory (Section 6) and distributed (Section 7) parallelization. Special emphasis here is put on the interplay of dependencies with spacetree traversal strategies as well as the programming interface. Notably, we discuss two competing multiscale data splitting strategies, synchronizationavoiding application interfaces, and a constraint technique that can guide a task-based parallelization. Some experiments highlight properties of the proposed realization ideas before an outlook closes the discussion.

\section{SPACETREE DEFINITION AND ENUMERATION PARADIGMS}

This article stands on the shoulders of spacetrees as a generalization of the quadtree/octree idea (Bader 2013; Weinzierl 2009; Weinzierl and Mehl 2011). The computational domain is suitably scaled and dilated to fit into a unit hypercube of dimension $d$. All ingredients introduced in this article as well as the underlying software work for any dimension $d \geq 2$. We cut the hypercube into $k$ equidistant slices along each coordinate axis and end up with $k^{d}$ small cubes. They form a Cartesian grid while the cut process describes a relation $\sqsubseteq_{\text {child of }}$ between the newly generated children and their parent cube. It yields an embedding of the finer into the coarser grid. We continue recursively yet independently for each new cell and end up with a set of cubes $\mathcal{T}$, where the tree leaves, i.e., the cells not refined further, span an adaptive Cartesian grid. The parentchild relation $\sqsubseteq_{\text {child }}$ of or its inverse, respectively, induce a hierarchy on $\mathcal{T}$. It renders the data structure into a tree. The overall construction process yields a cascade of disconnected Cartesian grids. They are embedded into each other but each individual resolution's Cartesian grid might be disconnected and not covering the whole domain. An "embed disconnected Cartesian grids" formalism is widely used among tree codes in fluid dynamics (Bungartz et al. 2010b; Deiterding 2005; Dreher and Grauer 2005; Dubey et al. 2016) - notably if they rely on the classic Berger-Colella patch language. Prior to this, early work on adaptive multigrid methods already relies on such data structures (Brandt 1973, 1977; McCormick 1989). While geometric multigrid and -level algorithms benefit from the nested grids, applications requiring only the finest tessellation ignore the coarser levels. 

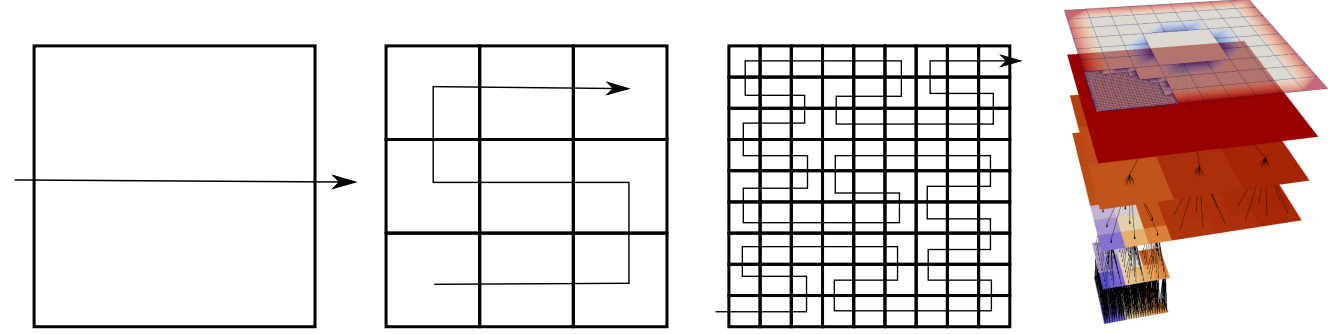

Fig. 1. We embed the computational domain into a square (left). The Peano SFC motif (second from left) induces an ordering on a Cartesian grid (next) that results from successive refinement starting from the square (left). Illustration following (Weinzierl 2009). The sequence depicts three regular grids $\Omega_{h, 0}, \Omega_{h, 1}$ and $\Omega_{h, 2}$, which can be embedded into each other. The construction of an adaptive Cartesian grid (right, top layer) yields a spacetree as visualized in the layer below (from Weinzierl et al. (2014)).

Two classic enumerations of $\mathcal{T}$ follow depth-first search (DFS) and breadth-first search (BFS). Yet $\sqsubseteq_{\text {child of }}$ plus DFS or BFS remain partial orders as long as no enumeration is defined on the children of the refined cells. If we use one leitmotif for all parent-children relations, then we end up with a space-filling curve (SFC) (Bader 2013). If properly equipped with rotations and mirroring, then the leitmotif yields the Hilbert or Lebesgue curve for $k=2$ or the Peano curve for $k=3$. In our code base, we rely on a Peano curve (Figure 1), which motivates our choice of three-partitioning $k=3$.

ObSERVATION 1. Tree and grid language for spacetrees are equivalent once we read an adaptive Cartesian grid as composite of disconnected regular Cartesian grids.

Let the level $\ell$ of a tree's node equal the length of the path to the root of the tree, i.e., the original bounding box. The nodes of one level span a grid

$$
\Omega_{h, \ell}=\{c \in \mathcal{T}: \operatorname{level}(c)=\ell\} \quad \text { with } \quad \Omega_{h}=\bigcup_{\ell} \Omega_{h, \ell} .
$$

Design Decision 1. Cells and vertices are made unique through their position and space plus the level. Thus, multiple vertices may coincide spatially but "live" on different levels.

Our approach offers complete level grids $\Omega_{h, \ell}$ and thus permits applications to hold data on each level separately. Multigrid and multiscale algorithms, for example, can exploit this fact (Reps and Weinzierl 2017; Weinzierl and Weinzierl 2018; Weinzierl et al. 2016). If an application exploits solely the finest grid $\Omega_{h}$, then vertices and cells are held redundantly and the design decision imposes an overhead. Coarse, "spatially redundant" data then are simply ignored. The overhead is bounded by a factor of $\sum_{\ell=0}^{\infty}\left(\frac{1}{k^{d}}\right)^{\ell}=\frac{1}{1-k^{-d}}$. All aforementioned approaches working with embedded Cartesian grids-the present software historically is inspired by the cited multigrid work-conceptionally hold partially spatial redundant data.

Given our multilevel grid representation, we define the term hanging vertex in a multilevel sense, too: Most vertices of a level have $2^{d}$ adjacent cells on the same level. Some vertices have fewer adjacent cells. They are hanging. Multiple hanging vertices from different resolution levels may coincide.

Any SFC-based DFS or BFS ordering linearizes the spacetree (Sundar et al. 2008). It enumerates all cells of the tree. Peano's core routines never use this enumeration explicitly. Instead, the enumeration orders all data structures used and thus is exploited implicitly. Yet one data administration approach built on top of the core routines uses explicit numbering. 
Within the total DFS order, cell numbers (identifiers) can be derived from the whole path from the root to any cell. The root has entry 0 . Children of a refined node inherit the node's identifier and append a number along the SFC-similarly to adding additional digits after the decimal point if numbers were taken from $[0,1]$. This way, it is possible to derive a cell's position and size from its identifier and to search the linearization of neighboring cells. The code formalism is equivalent to a formalization of a recursive function running DFS through the tree: Each call stack entry then implicitly encodes one edge within the tree graph from the spacetree's root to the current spacetree node. For a plain, continuous numbering of cells along the natural numbers this is not possible. Efficient numbering constrains the tree depth (Grandin 2015; Sampath et al. 2008) and stores each cell identifier with a fixed number of bits. DFS and BFS yield the same cell ordering if an algorithm solely works with $\Omega_{h}$ and neglects/omits coarse levels. SFC codes implicitly encode through their code length that multiple grid entities may coexist on different resolution levels, even though a user might decide not to store data on coarse levels overlapping finer resolutions (cf. application data storage in Section 4).

Design Decision 2. We use tripartitioning instead of the predominant bipartitioning, and we use the Peano space-filling curve $(k=3)$.

While Hilbert and Peano yield face-connected DFS enumerations, i.e., any two subsequent cells along the SFC enumeration share one face, only Peano and Lebesgue are straightforward to extend from $d=2$ to $d \geq 3$. See, for example, Bader (2013) for curve illustrations and discussions of their properties; Haverkort (2016) and citations therein for further formalization, classification, and variants; as well as Skilling (2004) for a high-dimensional generalization of Hilbert with links to Gray codes. Lebesgue uses a tensor-product approach, Peano extends its motif from $d$ into dimension $d+1$ by mirroring the $d$ motif once, appending it along the dimension $d+1$, and then appending on top the original motif again.

As we stick to Peano, our software uses $k=3$. The concepts of this manuscript, however, work for any $k \geq 2$. Our code base supports any $2 \leq d \leq 7$. $d \geq 8$ requires manual extension of a few macros, though it runs into the curse of dimensionality (Bellman 1961).

\section{SPACETREE APPLICATION PROGRAMMING INTERFACE}

AMR code interfaces can be classified along various metrics. We use two (Table 1). Both assume that the most important operation on an AMR grid is to run over all entities. An interface either can permit the user code to arbitrarily navigate through the spacetree, or a code can prescribe the traversal order of the grid entities. Orthogonal to this decision, a user interface has to define which data the user is allowed to read and write. Strict element-wise traversals allow a code to access solely the cell data themselves plus data of its adjacent vertices while they march through the grid. The other extreme of a cell-based API allows an application to read and write any data transitively associated to a cell: A code can process adjacent vertices of a cell, any neighboring cell associated through a face, or adjacent vertices of the neighbors, and so forth. The latter scheme supports, from the traversal's point of view, random access to the memory (RAM).

Design Decision 3. Peano sticks to a strict element-wise multiscale tree traversal. All cells of each tree level are processed per grid sweep. The process order is determined by the tree traversal code. The user has no influence on this order and has to program agnostic of it. However, many temporal constraints are guaranteed, i.e., there is a guaranteed partial order on the traversal's transitions. At any time solely cell data, the vertices adjacent to a cell, the cell's parent plus the adjacent vertices of the parent are exposed to the application code. 
Table 1. A Classification of Spacetree User Interfaces at Hands of Two Orthogonal Metrics

\begin{tabular}{l||l|l}
\hline & $\begin{array}{c}\text { restrictive, constrained (cellwise) } \\
\text { data access }\end{array}$ & $\begin{array}{c}\text { read and write of (non-local) grid } \\
\text { data (RAM) }\end{array}$ \\
\hline \hline $\begin{array}{l}\text { prescribed grid } \\
\text { traversal order }\end{array}$ & \multicolumn{1}{||}{ Peano } & $\begin{array}{l}\text { Examples are deal.II or Dune (with } \\
\text { user-defined large grid overlaps) if } \\
\text { solely iterators are used. Yet they } \\
\text { allow users to navigate through } \\
\text { the grid starting from a iterator } \\
\text { position ... }\end{array}$ \\
\hline $\begin{array}{l}\text { user controls grid } \\
\text { run-through order }\end{array}$ & $\begin{array}{l}\text { p4est, e.g., supports arbitrary } \\
\text { run-through orders while its ghost } \\
\text { layer of width one constrains/ } \\
\text { localizes data access. If users use } \\
\text { into each other. The exact } \\
\text { classification of both examples } \\
\text { depends on how the iterators are } \\
\text { code fits into the rubric above. }\end{array}$ \\
\hline
\end{tabular}

A restrictive programming model where the user is not in control of the access order allows us to hide how the data are held and maintained. It is thus our method of choice for a separation-ofconcerns software architecture.

\subsection{Supported Application Types}

Different applications fit to our strict element-wise traversal. We detail some applications in the appendix and summarize the key characteristics here. Naturally, any stencil that decomposes additively over $2^{d}$ cells arranged in a cube can be realized. $d$-linear finite element codes fall into this class as well as low-order finite volume and finite-difference schemes. We may assemble system matrices explicitly through PETSc (Balay et al. 2016), e.g., or make the grid traversal realize matrixfree matrix-vector products (Reps and Weinzierl 2017; Weinzierl and Weinzierl 2018). Arbitrary adaptivity is supported. We can code it recursively by writing two-level interaction operations. As Peano holds all grid levels, also different stencil types or even PDEs can be hosted by different resolutions.

A spacetree cell can host a whole patch of unknowns rather than only single unknowns. The same concept allows us to host higher-order shape functions. In this context, it is reasonable to weaken the notion of element-wise: If we augment, similarly to Khokhlov (1998), a vertex by pointers to its $2^{d}$ adjacent cells, then we can construct the inverse of the directed connectivity graph constructed by the spacetree. Through the vertices, a cell then has access to its neighbor cells or an adjacent coarser cell if there is no neighbor on the same level. Inter-grid transfer operators still can be realized through the traversal events.

Design Decision 4. By default, Peano offers strict element-wise multilevel data access. Yet we allow to weaken these data access permissions: At the price of $2^{d}$ pointers per vertex, we make each vertex point to its adjacent cell data.

This additional adjacency information is maintained, also for dynamically adaptive grids, by the traversal code: There is memory overhead to hold adjacency properties that allow us to weaken 

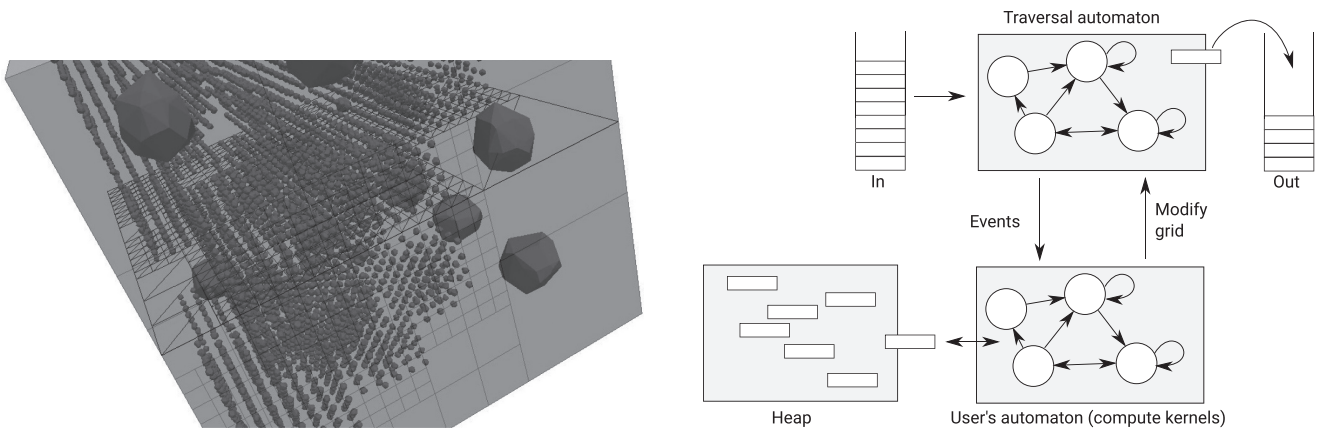

Fig. 2. Left: Snapshot of a Discrete Element code where rigid particles are embedded into the Peano grid. Right: The traversal automaton runs through the grid (top) reading in streams and piping out streams. Each transition triggers kernels in the application-specific automaton (bottom) that may use or may not use heap data.

the strictness of the term cellwise. There is no additional algorithmic cost as all links, also for hanging vertices, are kept consistent on-the-fly. ${ }^{1}$

Patch-based codes (block-structured AMR) can either fill ghost layers with copied data from neighbors or make a patch interact directly with neighbor patches (Weinzierl et al. 2014). If we embed $n \times n$ or $n \times n \times n$ or, in general, $n^{d}$ patches into each cell, then we can through the inverse adjacency information equip each patch with a ghost layer of a width of up to $n$. We come back to the impact of patches on runtimes in 8.1. Besides low-order ansatz spaces and patches, higherorder discretizations fit to the traversal as long as their support is localized. Classic Discontinuous Galerkin, where the shape function support covers solely one cell, yields admissible stencils. Here, multiple degrees of freedom have to be assigned to vertices or edges-edge unknowns always can be mapped onto vertex locations, which implies that solely vertices have to be kept persistent from a data structure point of view-or degrees of freedom have to be stored within the cell. Higherorder approaches with increased global smoothness are more difficult to realize: B-spline shapes spanning multiple cells, for example, seem not to fit the element-wise concept. Yet we may use $n^{d}$ patch data structures with a ghost layer of width $n$ carrying higher-order shape functions such as B-splines of order $2 n-1$. Support overlaps from one spline with others then can be evaluated.

Particle-grid formalisms such as Particle-In-Cell (PIC) fit to our concept as long as the particlegrid interactions do not span more than one cell: If particles fall into a cell, then they may interact solely with the $2^{d}$ adjacent vertices of this cell. Alternatively, we can assign each particle to its closest vertex and thus store them in a dual tree grid (Weinzierl et al. 2016). This facilitates wider interaction areas.

Particle-particle interactions are straightforward to realize if we rely on the aforementioned neighbor cell links (Eckhardt et al. 2015) and if the interaction radius fits the mesh width. Again, particles can live on different spacetree levels (Figure 2). Moving them through various levels facilitates tunneling where particles move more than one cell per time step (Weinzierl et al. 2016).

\subsection{An Automaton-based Traversal}

As we disallow the user to navigate through the spacetree herself (Table 1), we read the multiscale Cartesian grid traversal as a deterministic push-back automaton-the formal equivalent to

\footnotetext{
${ }^{1}$ The augmentation of the grid with pointers to neighbor cells as well as the particle (-in-cell) management are available as optional Peano extensions.
} 


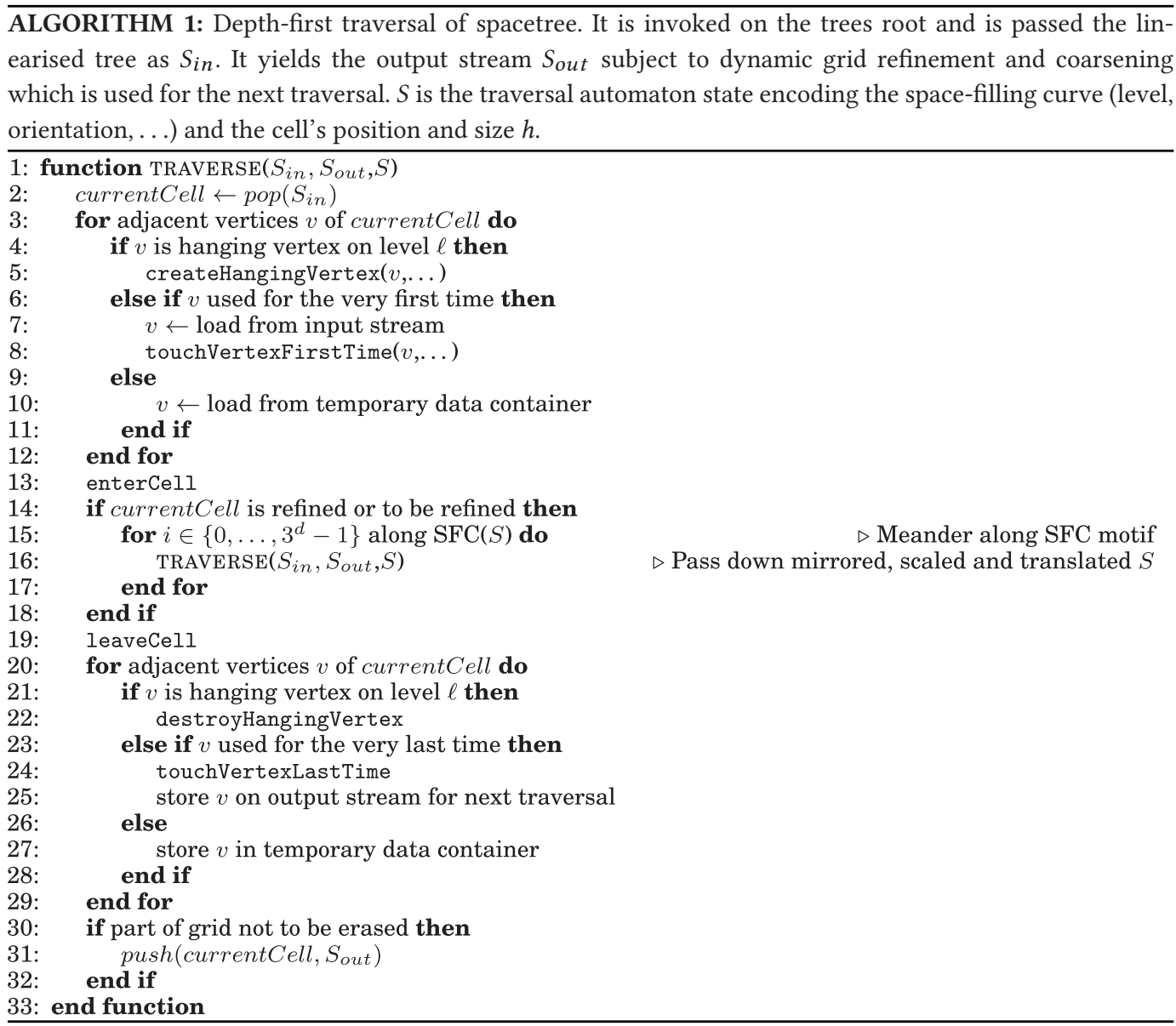

a recursive function-that reads the tree structure from an input stream while adaptivity criteria insert or remove elements. It runs from cell to cell (Algorithm 1).

Without the optimizations from Section 6, we make the automaton run through the tree along a modified DFS. Such a convention facilitates a memory-efficient realization of the automaton (compare Section 4) and makes the automaton run through each spacetree cell twice: once throughout the steps down and once when it backtracks bottom-up. This equals an element-wise multiscale adaptive Cartesian grid traversal that is formalized by a sequence of transitions such as "move from one cell into another cell." In our code, these transitions act as plug-in points for the applicationspecific functions (compute kernels) (Meister et al. 2012). We refer to them as events (Table 2). Events are defined on cells, vertices, or traversal start and end.

Design Decision 5. Peano does not impose any balancing condition. Each hyperface in the grid may host an arbitrary number of hanging vertices (Isaac et al. 2012; Sampath et al. 2008; Sundar et al. 2008). Balancing can be added optionally as an extension, i.e., without the need to code it manually (Appendix C).

A user writes routines that are invoked on grid entities through the transitions. The routines' semantics may depend on a state. Such a system can be read as a combination of two automata 
Table 2. Table of Events Defined by Peano That Act as Plug-in Points for the Application

\begin{tabular}{|c|c|}
\hline Event & Semantics \\
\hline $\begin{array}{l}\text { beginIteration } \\
\text { endIteration }\end{array}$ & $\begin{array}{l}\text { Is called once per tree traversal prior to any other event. } \\
\text { Is called once per tree traversal in the very end. }\end{array}$ \\
\hline createVertex & $\begin{array}{l}\text { Creational event (Gamma et al. 1994) that allows proper intialisation } \\
\text { of vertices. Is invoked only once per vertex if the grid is refined by } \\
\text { the traversal automaton. }\end{array}$ \\
\hline destroyVertex & $\begin{array}{l}\text { Counterpart of createVertex invoked just before the memory of a } \\
\text { vertex is released. Is invoked by the traversal automaton once before } \\
\text { it erases parts of the spacetree. }\end{array}$ \\
\hline createHangingVertex & $\begin{array}{l}\text { Hanging vertices are never held persistently but (re-)created } \\
\text { on-the-fly whenever they are required, i.e., whenever an adjacent cell } \\
\text { on the respective level is traversed. This implies that a hanging } \\
\text { vertex might be created up to } 2^{d}-1 \text { times. }\end{array}$ \\
\hline destroyHangingVertex & Counterpart of createHangingVertex. \\
\hline reateCell & Creational event for cells. \\
\hline destroyCell & Counterpart of $\mathrm{cr}$ \\
\hline touchVertexFirstTime & $\begin{array}{l}\text { Event invoked on a vertex once per traversal just before it is used for } \\
\text { the very first time. }\end{array}$ \\
\hline touchVertexLastTime & $\begin{array}{l}\text { Event invoked for a vertex after all adjacent cells have been } \\
\text { traversed. }\end{array}$ \\
\hline enterCell & $\begin{array}{l}\text { Whenever the traversal automaton enters a spacetree cell, it invokes } \\
\text { an enterCell event. }\end{array}$ \\
\hline leaveCell & $\begin{array}{l}\text { Counterpart of enterCell that is invoked throughout the } \\
\text { automaton's backtracking. }\end{array}$ \\
\hline descend & $\begin{array}{l}\text { Variant of enterCell that is offered to simplify multigrid algorithms. } \\
\text { Passes a refined cell plus its adjacent vertices to the } \\
\text { application-specific code as well as all } 3^{d} \text { child cells and their } 4^{d} \\
\text { vertices. }\end{array}$ \\
\hline ascend & Counterpart of descend. \\
\hline
\end{tabular}

(Figure 2): One runs through the spacetree. Its transitions trigger stimuli that feed the other automaton implementing the solver's behavior as reaction. Stimuli comprise both automaton properties such as position in space, level, grid statistics (such as the total number of vertices), and the data associated to the event. Data circumscribe vertex attributes for vertex-based events, cell attributes plus all attributes of adjacent vertices for cell-based events, and always the same type of data associated to the next coarser level, i.e., to the parent grid entity. The latter facilitates the realization of multiscale applications. Level and spatial properties are held within the automaton state and thus are not stored within the vertices and cells. This is memory efficient. The event-based programming model forces the user to express all algorithms in local operations, i.e., element-wise or per vertex with both having access to respective local coarse-grid counterparts. The application code may express the wish to refine or coarsen the grid to the traversal through the return value of the events.

Let $a, b \in \mathcal{T}$ with $a \sqsubseteq_{\text {child of }} b$. Further, $v_{a}, v_{b}$ are vertices adjacent to $a$ or $b$, respectively. Finally, $\sqsubseteq_{\text {pre }}$ is a temporal relation, i.e., clarifies which steps run in which partial order. While the 
processing order of cells and vertices is hidden, all applications can rely on the invariants

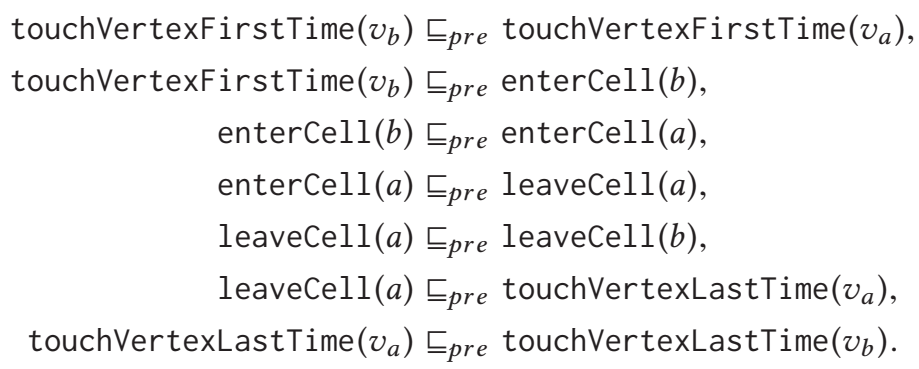

It identifies which event is invoked prior to another event. It is easy to verify that DFS and BFS both suit (1).

Design Decision 6. We allow the application automaton to flag operations from Table 2 as empty. They then are automatically skipped and thus removed from (1).

OBSERVATION 2. In the context of trees in object-oriented languages, our inversion of controlthe application kernels do not determine how the data structures are processed-can be read as a composite pattern in combination with a visitor pattern (Gamma et al. 1994). In the context of finite element (FEM) solvers, this paradigm is used by various codes (Burstedde et al. 2011; Meister et al. 2012; Weinbub et al. 2014). In the context of general programming, it mirrors higher-order functional programming where the application's function set is passed to the traversal function.

We favor to call the programming pattern Hollywood principle (Sweet 1985): Don't call us, we'll call you. The user's implementation is unaware of when events are invoked-though the constraints (1) hold-it is unaware where events are invoked in a distributed environment, and it is unaware of any other events invoked concurrently.

\subsection{Limitations of the Approach}

Our API poses limitations. First, the grid's structuredness does not offer the flexibility of unstructured meshes though users can weaken the structuredness by embedding unstructured/point data into the spacetree cells. Second, the hiding of traversal details makes the code not a black-box library that can be used within any code base through few library invocations. It requires users to break down their algorithm's workflow into events and dependencies first. The "loss of control" might require algorithm designers to rethink code snippets and algorithmic realizations. Finally, a very strict element-wise mindset is not the standard mindset of many application developers. This steepens the learning curve.

\section{PERSISTENCY MODEL AND DATA MANAGEMENT}

How to store the spacetree is an important design decision for software. Also, we have to clarify how to hold data associated to the grid.

\subsection{Spacetree Storage Schemes}

To hold the spacetree, we distinguish two paradigms: The tree either is mapped onto a graphtypically structures/classes with pointers-or it is held linearized. Hybrids exist. Graph-based approaches are flexible but suffer from pointer overhead. Furthermore, the tree records scatter in memory and induce non-local memory accesses for a traversal code once the grid is subject to strong dynamic adaptivity. This may cause poor memory usage profiles. Yet a spacetree mapped onto a graph data structure still yields a lower memory footprint than arbitrary unstructured grids, 
as associativity between cells is encoded in the tree relations: A cell neighbor either is a sibling or a particular child of the sibling of the parent node, and so forth. We note that some graph approaches exploit structuredness, i.e., combine the graph with linearization: Siblings can be stored as one block (Gadeschi et al. 2015; Khokhlov 1998) or regular grids are used on certain grid levels (Feichtinger et al. 2011) and thus increase data access locality while they reduce the memory footprint.

As alternative to the graph, we may hold the spacetree in a stream. This stream holds all cells of the spacetree according to a total order on $\mathcal{T}$. A natural approach stores the DFS-plus-SFC code within the stream (Sampath et al. 2008). As the code comprises the cell's level plus position, lookups for neighbors within the stream are efficient-a neighbor's code can directly be computed by bitwise index manipulations. If BFS determines the total storage order, then the stream consists of chunks. Each holds data from one tree level (Bangerth et al. 2011, 2007). If DFS determines the total storage order, then the individual resolution levels are interwoven, i.e., vertically integrated (Adams et al. 2016a).

OBSERVATION 3. Linearized spacetrees are superior to graph-based data structures, i.e., structs connected via pointers, in terms of memory as they only require one code (identifier) to be stored per cell.

As we forbid control over the traversal order in our code and stick to a DFS/SFC combination, we can implement the tree traversal as recursive function (Algorithm 1). It is a push-back automaton relying on the system's call stack. The automaton knows at any time its level and position and thus solely has to know from an input linearization whether to recurse further or not. No maximum tree depth constraints are imposed while two bits per cell (unrefined, refined, to be refined, to be erased) are sufficient to store all the dynamic adaptivity information (Weinzierl and Mehl 2011). For static adaptive grids, one bit is sufficient. In Algorithm 1, these bits are encoded within $S_{i n}$.

Design Decision 7. Peano linearizes the tree along the Peano SFC. It sticks to a DFS, and the traversal thus reads in the spacetree as bit stream.

To support arbitrary adaptivity, it is advantageous to make the automaton read one bit stream and output another stream that acts as input to the subsequent traversal. We hence avoid data movements due to insertion and deletion while the stream read/writes are advantageous in terms of memory access characteristics. They yield high temporal and spatial data access locality (Kowarschik and Weiß 2003). Yet for reasonably structured grids we abandon the strict linearization (Section 5).

\subsection{Application Data Management}

Automaton-based spacetree traversals do not prescribe how the actual application records are held. Besides graph-based descriptions-spacetree entities hold pointers to cell or vertex properties-two storage strategies are convenient. On the one hand, spacetrees can be both a technique to encode the grid structure and a container for the data themselves. On each and every level, the user assigns data to vertices and cells, and the stream of the spacetree is enriched with this application-specific data. The spacetree then acts as both organizational and compute data structure. On the other hand, we can rely on a heap. All data are stored within a (hash) map. The cell's DFS/SFC identifier contained in the automaton state is a natural candidate to provide a key to this hash map: If we compute the hash code from both the spatial position along the SFC plus its level, then dynamic mesh refinement does not require frequent hash map reordering or induces many hashing conflicts.

Alternatively, we may use pointers from grid entities to map entries. With a semantic separation of the tree/grid data container from an application container, the realization resembles Bangerth et al. (2011). However, our implementations are originally inspired by Griebel and 
Zumbusch $(1998,1999)$. If the grid does not change too frequently, then it is, as a variation of the hash storage, convenient to flatten also the application data along the spacetree linearization and hold it as one big chunk of data accessed along the SFC. This is particularly interesting for hardware suffering from indirect and scattered memory access. We omit this variant for the heap as we assume that our grid changes in each and every grid sweep. Yet we also point out that embedding user data into the linearized tree stream, i.e., enriching the tree stream, delivers such data flattening/reordering implicitly.

Design Decision 8. Both data storage strategies-embedding data into the spacetree and holding it in a separate hash map-are available in Peano: We refer to them as stack-based or heap-based data storage.

Both variants come along with pros and cons. If we embed PDE-specific data into the read/write streams, then data associated to the respective cell as well as vertex data are immediately available to the automaton. Records associated to the spacetree cells are directly interwoven with the spacetree's bitstream. Records associated to the spacetree vertices are ordered along touchVertexFirstTime and are conveniently held in a separate stream. Yet they could be merged into the cell data structure. We show in Weinzierl (2009) and Weinzierl and Mehl (2011) that we can write out the vertex data as stream following touchVertexLastTime and use this stream as input the subsequent iteration even if the grid is dynamically adaptive. Throughout the grid traversal, vertices temporarily have to be stored on stacks. The stacks serve as temporary data containers in Algorithm 1.2 $d$ such stacks are required, and their maximum size is bounded by the spacetree depth. The number of stacks is fixed and small, and their size is small, too. Cell-vertex associativity is encoded in the grid traversal automaton, i.e., the automaton knows at any time from which data container (stream or stack) to take all vertex data from, and it knows where to write vertices to before the next cell is entered. The whole scheme comes for free in terms of user source code-the automaton simply hands over references to the stacks to the user automaton-the total memory footprint is minimal and all data remain read and written in a stream/stack fashion. This yields excellent memory access characteristics (Bungartz et al. 2010b; Mehl et al. 2006; Reps and Weinzierl 2017; Weinzierl 2009; Weinzierl and Mehl 2011; Weinzierl et al. 2016).

The method falls short if the data cardinality per vertex and cell varies-our cell or vertex stack entries all have to have the same byte count-or if the data per cell/vertex is massive and thus moving it from one stack to another is expensive. The former case covers the frequent situation that some data such as matrix entries are not required persistently over the whole simulation workflow. In this case, heap-based storage is advantageous though it requires additional coding, induces hash bookkeeping overhead, and may introduce scattered data with non-uniform data access cost. Yet efficient hash codes exist (Robey et al. 2013; Tumblin et al. 2015). Notably, DFS/SFC codes yield high-quality hash codes or preimages to a hash function due to their Hölder continuity (Bader 2013; Bungartz et al. 2006; Gotsman and Lindenbaum 1996; Griebel and Zumbusch 1998, 1999; Hungershöfer and Wierum 2002). If a mesh remains stationary or remeshing cost are negligible compared to the compute load, then it finally is convenient to make the "hash code" store all user data continuously in memory (Tu et al. 2005). The user data are linearised. We do not follow up on this option, as we focus on changing meshes.

\section{REDUCTION OF TREE TRAVERSAL OVERHEAD}

DFS tree traversals are a powerful tool to facilitate arbitrary dynamic adaptivity for meshes that change frequently. As we realize the tree's linearization through a recursive function, i.e., a pushback automaton, we, however, end up with a code that requires a certain amount of integer arithmetics and callstack administration (recursion overhead). This overhead is not negligible if (i) we 
study codes with low arithmetic intensity per grid entity and (ii) the user cannot mask out operations a priori. The latter feature is enabled by additional callbacks that can be used to clarify that enterCell, for example, in some algorithmic phases never is invoked on refined cells. In this case, Peano omits them automatically, and it obtains performance closer to codes that work on the fine mesh $\Omega_{h}$ only-though the performance gap never will be closed, as our code is inherently written for multiscale.

This cost can be reduced if we embed regular subgrids (patches) into the cells (Weinzierl et al. 2014). Traversing Cartesian arrays is among the best-understood and cheapest traversal variants. A code with a spacetree hosting regular patches thus yields an administrative overhead that is between the minimalist cost of Cartesian grids and the cost of unconstrained dynamically adaptive spacetree meshes. A refinement criterion then is not free to adopt arbitrarily accurate to a feature anymore. It has to overlap features of interest with patches. As alternative to patches, one can temporarily or locally disallow the tree to coarsen or refine and then skip many logical checks.

Uniform meshes are a special type of spacetree meshes: Let a balanced spacetree be a tree where any path from the root to a leaf has the same length. A balanced spacetree yields a cascade of regular Cartesian grids. We therefore refer to such trees as regular spacetrees. Their BFS traversal first running from coarse to fine and then backtracking from fine to coarse combines the efficiency of regular Cartesian access with the constraints from (1). We thus identify regular subtrees within the spacetree that do no change and do not accommodate any hanging vertices-they trigger additional events-on-the-fly and to switch from the DFS event invocation on this spacetree to BFS (Eckhardt and Weinzierl 2010).

\subsection{Transformation of DFS into BFS}

To find the regular subtrees Peano relies on an analyzed tree grammar (Knuth 1990). Let each spacetree cell hold a marker $f$ with

$$
\forall c \in \mathcal{T}: f(c)= \begin{cases}0 \quad c \text { is a leaf with no hanging adjacent vertices, } \\ \hat{f} \text { if } c \text { is refined and } \forall a \sqsubseteq_{\text {child of }} c: \\ \quad f(a)=\hat{f}-1, \text { or } \\ \perp \quad \text { otherwise. }\end{cases}
$$

$\perp$ marks spacetree nodes that root adaptive, non-regular subtrees or regular subtrees framed by hanging vertices. Equation (2) is accompanied by some veto mechanisms overruling the outcome of (2): If refinement or grid coarsening is triggered, then all markers of surrounding cells are cleared to $\perp$. $f$ is an augmentation of the spacetree bit stream and can be maintained on-the-fly: We take $f$ from the input stream for our optimization while we concurrently redetermine its new value for the subsequent traversal. In any iteration following the identification of an $f>1$, the traversal automaton can modify its event invocation and data processing (Eckhardt and Weinzierl 2010; Schreiber et al. 2013a): If it encounters a cell with label $f>1$, then it knows that from hereon a regular subtree of depth $f$ is to be traversed. We know how much data for this subtree are to be read from all input streams, and we create a temporary buffer in the memory than can accommodate the whole regular subtree as a cascade of regular Cartesian grids. This buffer is filled. Following the load, the automaton invokes all the touchVertexFirstTime, enterCell, and so forth, events in a BFS order before the tree is streamed to the stacks again. Formally, the reordering is local recursion unrolling.

If the BFS is fed a refinement or coarsening request by the application automaton, then the markers in the regular subtree are set to $\perp$, and we bookkeep the erase or refine request. It is 

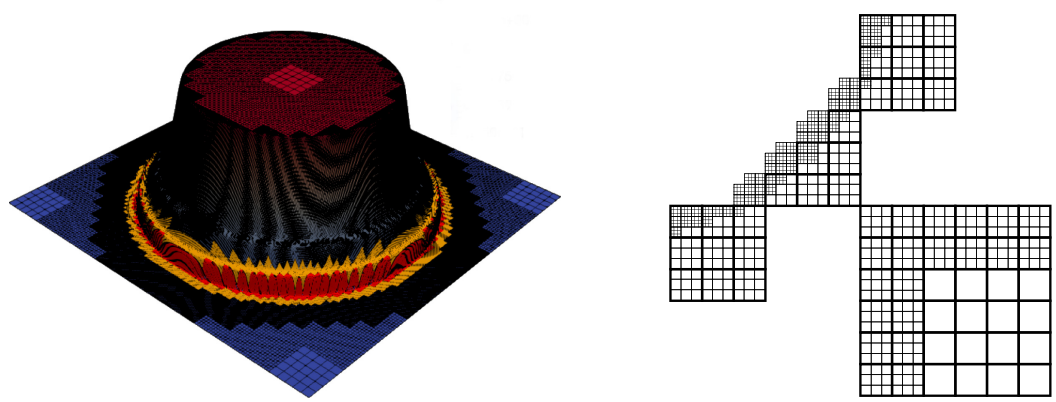

Fig. 3. Left: Euler equation simulation of a point shock from the ExaHyPE project (Bader et al. 2015). Right: Illustration of the skeleton grid of the bottom corner from the left simulation, i.e., the grid that is held as a tree if all regular subtrees of dimension $9 \times 9$ or bigger are held as separate regular grids.

realized in the subsequent iteration when the subtree is not treated as a regular one anymore due to the invalidated $f$.

\subsection{Persistent Regular Subtrees}

By default, the DFS-BFS transformation is applied locally, on-the-fly, and temporarily, while we preserve the DFS/SFC input and output order on all streams. Though our approach eliminates many case checks and allows for an efficient triggering of events, it retains recursive code parts for the loads/stores from the streams to the Cartesian buffers and back.

Provided that regular subtrees remain regular, we can remove them from the tree stream and hold them as cascade of Cartesian mesh separately (Figure 3). Vertices from the regular subtree that are adjacent to the remainder of the (adaptive) grid are replicated. Prior to entering a regular subtree, these vertices are updated with the most recent vertex version from the spacetree stream. Once a subtree is processed, vertices are mirrored back. These consistency updates can be done efficiently as they affect a lower-dimensional submanifold only and as a projection of the Peano SFC onto the face of the cube spanned by the regular subtree yields a dimension-reduced Peano SFC on the face again (Weinzierl 2009; Weinzierl and Mehl 2011). The projection yields a total order that matches the inverse of the SFC's total order on the remaining grid's entities (Schreiber et al. 2013a). Our linearized spacetree spans a holed grid, a skeleton grid, with links to regular subtrees. Though this is a pointer-linearization hybrid, consistency data exchange all follows the SFCs through stacks.

If an application code wants to modify the grid within a regular subtree, then we postpone the grid modification and first reintegrate the linearised subtree into the spacetree stream. The followup grid sweep then refines or coarsens. The technique follows the cluster-based AMR of Schreiber et al. (2013b) but combines it with (2) to identify stationary clusters on-the-fly, applies it solely to regular subtrees, and augments it by the multiresolution grid notion.

It is similar to approaches composing the AMR grid as assembly of regular patches. However, we do not start from regular patches as building blocks. Instead, we identify regular subregions on-the-fly and treat them then more efficiently than the remainder of the grid. Therefore, the adaptivity of the spacetree mesh that can be constructed is not restricted at all (Table 3). Regular subgrids are held separately and the linearised tree encodes only the skeleton in-between or those regions that change frequently (Figure 3). The grid is decomposed. We notice the similarity of this decomposition to a generalized variant of enclave partitioning (Sundar and Ghattas 2015) though we use our technique solely to reduce administrative overhead rather than to parallelize. 
Table 3. One Characterization of Various Levels of Structuredness and Flexibility for Locally Regular Adaptive Grids

\begin{tabular}{l|l|l|l}
\hline & $\begin{array}{l}\text { Block-structured } \\
\text { AMR (patch-based) } \\
\text { with logical tree } \\
\text { topology between } \\
\text { patches }\end{array}$ & $\begin{array}{l}\text { On-the-fly block } \\
\text { identification as } \\
\text { realized in Peano }\end{array}$ & Pure tree codes \\
$\begin{array}{l}\text { Regular Cartesian grid } \\
\text { Globally simple, } \\
\begin{array}{l}\text { administration and } \\
\text { data access }\end{array}\end{array}$ & $\begin{array}{l}\text { Simple per entity } \\
\text { (block/ptach) }\end{array}$ & $\begin{array}{l}\text { Simple per identified } \\
\text { block; remaining } \\
\text { (skeleton) grid complex }\end{array}$ & $\begin{array}{l}\text { No regular data } \\
\text { accesses and simple } \\
\text { programming } \\
\text { (without indirect } \\
\text { addressing, e.g.) }\end{array}$ \\
\hline No adaptivity & $\begin{array}{l}\text { Adaptivity } \\
\text { constrained }\end{array}$ & $\begin{array}{l}\text { No constraints on } \\
\text { adaptive patterns }\end{array}$ & $\begin{array}{l}\text { No constraints on } \\
\text { adaptive patterns }\end{array}$ \\
\hline
\end{tabular}

While the simplicity reduces form left to right, the flexibility (where are we allowed to invest grid entities) increases.

\section{SHARED MEMORY CONCURRENT TRAVERSALS}

With dynamically adaptive grids, shared memory parallelization shall be lightweight (i.e., avoid expensive setup phases), shall not synchronise much data, and shall enable work stealing to adapt seamlessly to workload changes. Task-based systems promise this.

OBSERVATION 4. Our event-based programming model can be casted into a task language: the user implements a fixed set of task types (event implementations), the constraint set (1) describes task dependencies, and the tree instantiates the tasks.

\subsection{Dependency-based Programming Interface}

The observation implies that we may throw any pair of traversal and user automata directly into a task management system. However, not all codes allow for a concurrent invocation of all events from (1). They impose additional constraints on the data accesses.

A matrix-free matrix-vector product, for example, may not allow two adjacent cells on the same level to add their residual contributions to the vertex concurrently. Red-black Gauß-Seidel-type colouring of cells with $2^{d}$ colours ensures that no vertex is accessed simultaneously in this case. Peano allows user codes to specify per event per algorithmic step which colouring would ensure on a regular Cartesian two-resolution grid that no data races occur. We support a range of colouring choices:

- A complete serialization that is, for example, important to events that run IO and thus have to veto any concurrency.

- A degenerated colouring (one colour) implies that all cells or vertices, respectively, can be processed concurrently.

$-2^{d}$ colouring of cells ensures that no two cells access a shared vertex simultaneously. This implies that no face between two cells is accessed simultaneously by its two adjacent cells.

$-3^{d}$ colouring on cells ensures that no two cells with the same parent cell are handled concurrently. This is useful for multiscale algorithms.

$-4^{d}$ colouring generalizes this idea to vertices.

$-6^{d}$ colouring ensures that whenever two cells are handled in parallel, and their parent cells do not share any vertex.

$-7^{d}$ colouring generalizes this idea to vertices. 
Table 4. If Ran on Shared Memory, Peano Introduces Two Additional Events

\begin{tabular}{l|l}
\hline Event & \multicolumn{1}{c}{ Semantics } \\
\hline Copy constructor & $\begin{array}{l}\text { The class holding all events, i.e., the automaton, is replicated in } \\
\text { parallel sections per thread. The copy constructor allows the user } \\
\text { to plug into the replication. } \\
\text { If the traversal automaton leaves a grid region handled } \\
\text { concurrently, then all adapter replicates are merged into a master } \\
\text { copy and destroyed afterward. This merger is realised as tree } \\
\text { reduction as found in Intel's Threading Building Blocks (TBB). }\end{array}$ \\
\hline
\end{tabular}

DESIGN DECISION 9. We allow the user code to specify per event which concurrent data writes have to be avoided on a regular grid. It is Peano's responsibility to schedule a well-suited parallel execution of all tasks.

For a given spacetree $\mathcal{T}$, the constraints (1) plus the colouring per event define a race-free task graph. While we ask the user to model colouring constraints in terms of a cascade of regular grids and hide the complexity of multicore processing of a tree, all constraints translate to the dynamically adaptive grid.

\subsection{Task Parallelization without Task Graph Assembly}

Once a task graph is determined, there are two main possibilities to issue the tasks: The task graph can be assembled and handed over to a scheduler. For static grids, this can be done in a preprocessing step. For dynamically adaptive grids, it has to be done once per grid sweep, and the obtained concurrency has to make up for assembly cost. Alternatively, a grid traversal order that accommodates the task graph can be chosen: Continuous chunks of grid entities within the traversal order describe independent tasks and can be in parallel before the next chunk of grid constituents is traversed.

Design Decision 10. In Peano, the traversal order follows the task dependencies and the task dependency graph is never set up explicitly.

We notice that the DFS chosen for the spacetree linearization (Algorithm 1) exhibits a poor concurrency. All cell accesses are serialized. Solely a few vertex-based events such as touchVertexFirstTime can be evaluated in parallel. If the user code adds a dependency between these vertices, then even this negligible concurrency is eliminated. While we realize concurrent event invocations in the DFS traversal and also parallelize the automaton code itself, BFS is a better traversal for parallel fors/stencil codes.

We accept the DFS' limited concurrency in general and use the recursion unrolling's BFS to issue tasks in parallel if possible. Within regular, unrolled subtrees, we iterate through the levels and rely on parallel fors with colouring to issue user tasks in parallel. The colouring is guided by the user through her colouring choices describing data dependencies. Reordering of spacetree cells and the concurrent triggering of events impose bulk-synchronous programming (BSP) on the application automaton: The application normally is passed events sequentially. When the traversal automaton runs into a regular subtree, it forks the application automaton. The BFS event invocations subsequently are reordered to suit the dependencies. When the automaton leaves a regular subtree, all application automata are merged again. We reveal this by two additional events (Table 4) establishing an API that hides the realization with OpenMP or Intel's Threading Building Blocks. They allow the application developer to focus on data dependencies and data reduction. 

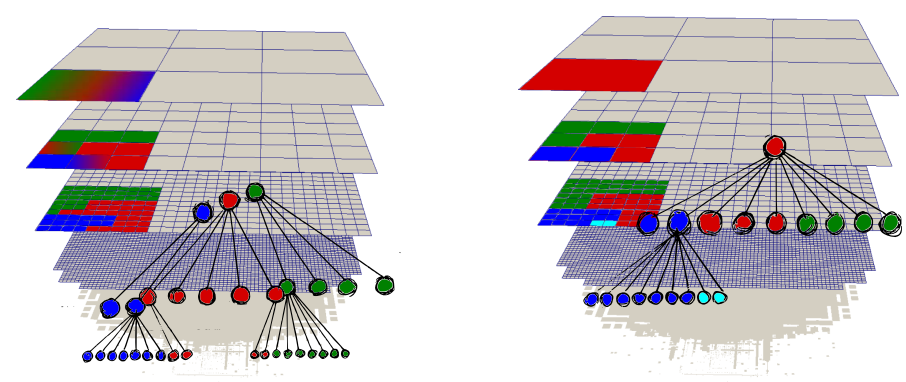

Fig. 4. Bottom-up spacetree decomposition (left) vs. top-down approach (right).

\subsection{Limitations of the Approach}

BSP as currently realized in Peano does not support data access patterns where some cells depend on their neighbours or parents while other cell pairs induce no constraint. We assume homogeneous dependencies. The parallelization kicks in for regular subgrids, while unstructured grid regions, i.e., the skeleton, the MPI domain boundaries or start and wrap-up phases of single grid traversals do not really benefit from multiple cores. If the grid changes globally per grid traversal or does not exhibit regular subregions, then our approach does not exhibit lots of concurrency. Our results uncover some of these effects. Finally, we note that sophisticated multithreading exploits concurrency spanning multiple iterative sweeps. Such a temporal blocking is not built in.

\section{TREE DECOMPOSITION}

We discuss our non-overlapping spacetree decompositions in this section. Non-overlapping refers to the finegrid here, i.e., we assume that each finegrid cell/spacetree leaf is assigned to exactly one rank. Extensions of our techniques to overlapping decompositions are beyond scope.

We emphasize that our goal is to retain the spacetree concept in a distributed environment. Despite the parallelization, we make each rank still hold a spacetree and, thus, we allow each rank to continue to traverse its spacetree as discussed so far. The traversal logic from a serial code (Table 2) is agnostic regarding the parallelization. However, there are additional MPI-specific events. They ensure that each cell is traversed only by one rank and that the constraints (1) are globally preserved, unless this is explicitly not required by the user code.

\subsection{Bottom-up and Top-down Tree Partitioning}

If the finest grid level is non-overlappingly distributed among ranks, then we have to clarify which rank holds which coarser grid entities. Two options exist (Figure 4): We can either assign the responsibility for every cell on every level to a unique rank or we can replicate coarser grid levels on multiple ranks. Such a distinction is important even for codes working solely with the finest grid, as it clarifies whether individual ranks are aware of the overall spacetree decomposition or just manage local decomposition knowledge. For the actual dataflow, however, both tree partitioning approaches become the same if solely the finest grid $\Omega_{h}$ is of interest.

Spacetree replication results from a classic bottom-up scheme (compare Bangerth et al. (2011), for example): We start from the adaptive finegrid and decompose its cells into chunks. From hereon, we construct each rank's spacetree recursively: Let a cell $a \in \mathcal{T}$ be held by a particular rank with

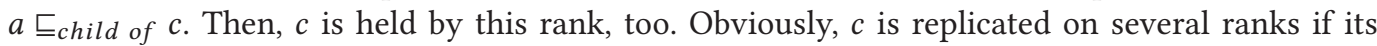
children are replicated or distributed among several ranks. The coarsest spacetree cell is available on all ranks. Bottom-up schemes often use the term local tree for the tree held on a particular 
rank. The coarser the level the more redundant the data. This implies that (fragments of) global knowledge about the chosen domain splitting is available per subtree.

The opposite approach without data replication is a top-down splitting (Weinzierl 2009): We assign the global spacetree root to one rank. For each $a, c \in \mathcal{T}, a \sqsubseteq_{\text {child of }} c$ with $c$ associated to a rank $r_{1}, a$ is either held by this rank $r_{1}$ as well, or it is deployed to another rank $r_{2}$ that has not been employed on a coarser or the same level yet. We exempt siblings along the SFC from the latter constraint. The rule applies recursively. It introduces a logical tree topology among the compute ranks: $r_{2}$ serves as worker to a master $r_{1}$. Whenever a child of a refined cell is assigned to another rank, this child acts as root of a remote tree. We cut out subtrees from the global spacetree.

ObServation 5. The majority of spacetree codes favor, to the best of our knowledge, the partial replication with SFC cuts.

Using SFCs to obtain an appropriate initial splitting of the finest grid level is popular. SFC partitions exhibit an advantageous surface-to-volume ratio ${ }^{2}$ and result from a straightforward splitting of the SFC's one-dimensional preimage, i.e., the enumeration of cells. We note that SFCs also can be used for the top-down splitting as the SFC motif orders all levels: As long as any rank forks subtrees only along the SFC to other ranks, the resulting splitting is an SFC-splitting, too.

Design Decision 11. Peano works with a non-replicating data layout, i.e., with the top-down tree partitioning. This tree distribution induces a logical master-worker topology on the ranks.

Ranks in Peano are aware only of their multiscale neighbors as well as their master and worker ranks. No global information is held per rank.

\subsection{Parallel Tree Traversal}

A parallel tree traversal on replicated trees yields one automaton per rank, each traversing its local linearised tree. All start their tree traversal at the same time. In this approach, tree nodes are labeled as replicated, local, or empty. Empty spacetree nodes are required if only some children of a refined node are processed locally, i.e., they are nodes purely required to complete the spacetree. As multiscale data are held redundantly, all information flow from coarser to finer grids can be realized without communication. After or throughout the backtracking, all replicated data have to be synchronized. Peano, however, works without replication.

A parallel tree traversal for the non-replicated tree yields one automaton per rank, too. However, the automata may not run in parallel right from the start, since they are synchronized with each other through (1). If a child of a refined cell is assigned to a remote rank, then the remote rank's traversal automaton is triggered to start traversing "its" tree by the automaton traversing the refined cell. This motivates the term worker. Throughout the bottom-up steps, an automaton in return has to wait for workers to finish prior to further backtracking. Both master-worker and worker-master communication are point-to-point communication keeping the two-grid interface between masters and workers consistent.

DFS is problematic for parallel codes without replication unless communication is explicitly eliminated as discussed below: It is strictly sequential. Peano therefore applies one-step recursion unrolling (Eckhardt and Weinzierl 2010; Weinzierl 2009) on the DFS: In each refined node, the automaton reads in all children. After the $k^{d}$ children are processed, they are put on the call stack,

\footnotetext{
${ }^{2}$ To the best of our knowledge, the good ratio is an empirical observation that can be backed-up by proofs only for regular grids where it results directly from the Hölder continuity (cf. Bader (2013) and Bungartz et al. (2006) and references therein). Further advantageous connectivity properties are discussed in Burstedde et al. (2017) and, notably, Haverkort (2016).
} 


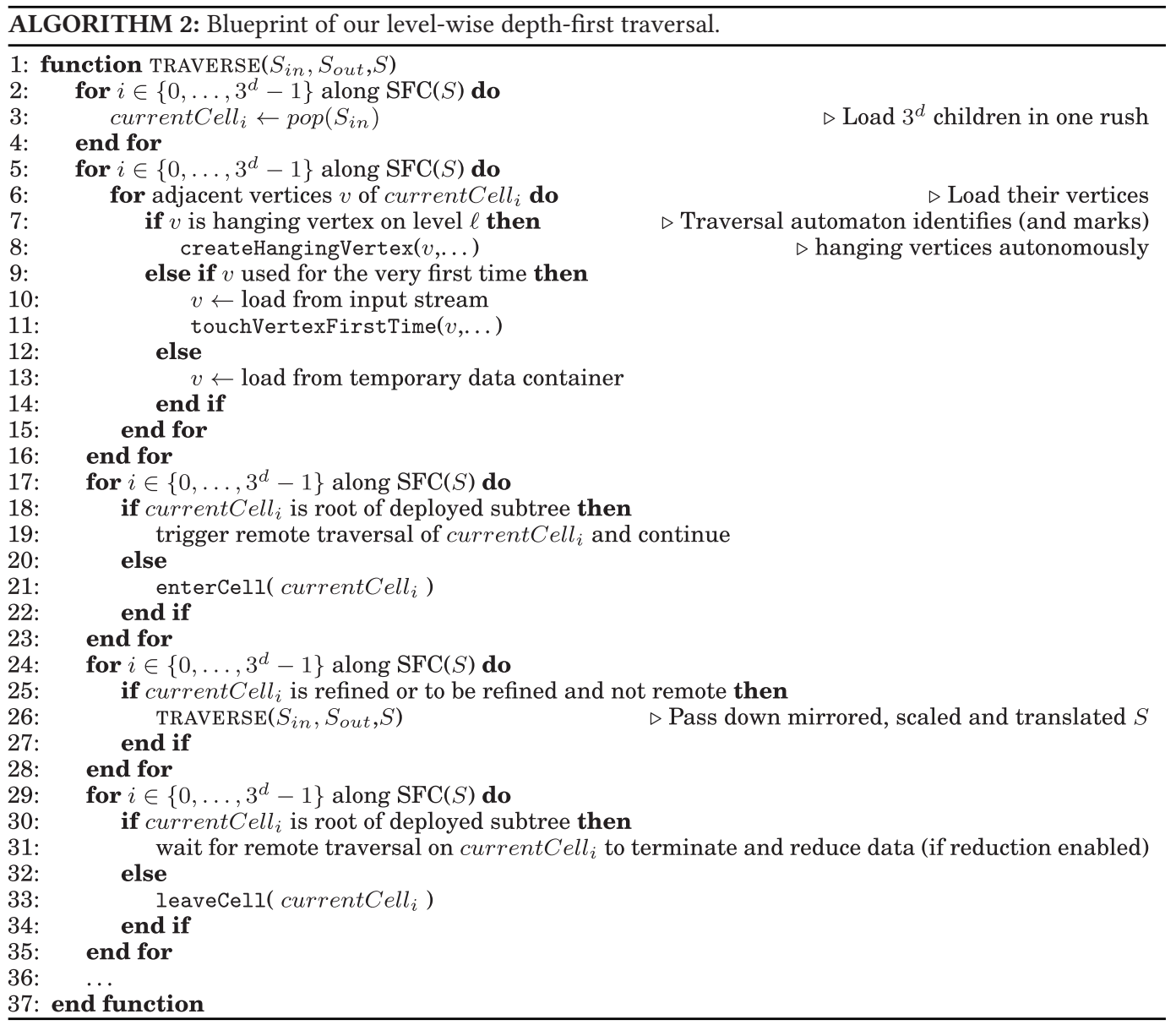

and the automaton descends along the children's order. Once all $k^{d}$ recursive descends have terminated, $k^{d}$ cells are taken from the call stack and the code backtracks. This is a one-step breadthfirst traversal within the depth-first framework. We call it levelwise depth-first (Algorithm 2). It allows us to trigger remote subtree traversals before we descend locally. Though it resembles (2), it does not rely on $f$ and is applied always; even if the tree is subject to change. Levelwise DFS is not literally a third traversal paradigm realized: It evolves from DFS through a one-step recursion unrolling, while BFS can be read as transitive hull of one-step recursion unrolling steps over DFS. Peano's mesh traversal thus can be formalized through DFS plus one-step recursion unrolling.

The replicating scheme comes along with a higher memory overhead than the top-down splitting. There is, consequently, more data to keep consistent, and data exchange involves typically more than two ranks-notably on the global root of which all ranks hold a replica. The top-down approach requires solely point-to-point data exchange and minimizes data redundancy. However, top-down induces a tighter, latency-sensitive coupling (Weinzierl et al. 2016): data of coarse cells are propagated into finer-grid resolutions that might act as coarsest resolutions to remote trees throughout the descent of the automaton. This on-the-fly information propagation has to integrate 
into the wake-up calls of traversal of worker ranks. Similar observations hold for the bottom-up information flow.

\subsection{Data Exchange}

Both decomposition schemes distinguish two types of data exchange: Vertices that are adjacent to cells handled by different ranks are replicated among all ranks and are subject to horizontal data exchange (Reiter et al. 2013) to keep them consistent. Vertices and cells that are held on two ranks due to a master-worker decomposition are subject to vertical data exchange.

Vertical data exchange is synchronously realized. Data are sent from the master to the worker upon the wake-up call, and coarse information from a traversal thus prolongs immediately, i.e., in the same traversal, to the worker. Data are sent from the worker back to the master when the worker terminates. Fine-to-coarse data propagate immediately in the same traversal.

Horizontal data exchange is asynchronously realized by non-blocking MPI. Vertex copies and their data are sent out from one rank to all other ranks holding a replica once they have been processed by the traversal. It is received prior to the first re-read of a vertex in the subsequent traversal. We therefore throttle the refinement with a marker-refine scheme: Ranks may trigger a refinement or coarsening, respectively, in one iteration.However, i is not realized along a parallel boundary before the subsequent traversal where all ranks holding a replica of a vertex have received the grid modification request. Both data exchange patterns apply to stacks and heaps.

The usage of the Peano SFC simplifies and speeds up the realization of the horizontal data exchange. Let $v_{a}$ and $v_{b}$ be two vertices held both on rank $r_{1}$ and $r_{2}$. We align the traversal orders on both $r_{1}$ and $r_{2}$ such that $v_{a}$ is used for the last time before $v_{b}$ on both ranks. Each rank thus can send out $v_{a}$ immediately to the other rank once $v_{a}$ has been used for the last time. The exchange of $v_{a}$ is automatically hidden behind the remainder of the traversal (finishing work on $v_{b}$, e.g.). In practice, multiple vertex sends are grouped into one chunk of data to reduce MPI overhead.

We reiterate that Peano's projection onto the surface of a partition yields a Peano curve of a reduced dimensionality (Weinzierl and Mehl 2011) and thus totally orders all vertices/faces on any subpartition. As a result, the data exchange between any two ranks can be modeled by one channel/ stream and no reordering of any incoming data is required as long as we invert the traversal on all ranks after each grid sweep (Bungartz et al. 2006). $v_{a}$ is sent out before $v_{b}$. The send of $v_{a}$ is hidden behind the treatment of $v_{b} . v_{b}$ is read in the subsequent iteration from the remote rank before $v_{a}$.

Design Decision 12. Peano exploits the fact that the Peano SFC projected onto a partition boundary yields a d-1-dimensional Peano SFC (Weinzierl and Mehl 2011). This makes all data exchange streams without any reordering. To stick to this paradigm, we disable all DFS-BFS transformations along MPI boundaries.

To realize this disabling, we set the markers in (2) for all cells along an MPI boundary to $\perp$. A hybrid MPI+X code runs MPI globally, the grid skeleton per rank remains single core, and it triggers shared memory parallelization only on interior enclaves (Sundar and Ghattas 2015).

\subsection{Parallel Programming Interface}

Peano's non-overlapping strategy with its logic tree topology is mirrored by additional events (Table 5). MPI Peano applications are strict extensions of a serial code base. No behavior of serial events is altered. The main control loop is run only on rank 0 (global master), and any choice of a particular event set to be ran is automatically broadcast to all working ranks. Upon an iterate, the global master's traversal automaton starts to run through its tree and recursively triggers traversals on all other ranks' spacetrees. Per rank the same event set is invoked. 
Table 5. Additional Events That Are Available in Peano If Code Is Compiled with MPI

\begin{tabular}{|c|c|}
\hline Event & Semantics \\
\hline mergeWithNeighbour & $\begin{array}{l}\text { Called for a vertex per neighbouring rank before } \\
\text { touchVertexFirstTime is invoked. Passes a copy, i.e., the } \\
\text { received replicate, from the neighbour alongside with the vertex } \\
\text { data. }\end{array}$ \\
\hline prepareSendToNeighbour & $\begin{array}{l}\text { Counterpart of mergeWithNeighbour that is called per } \\
\text { neighbouring rank after touchVertexLastTime to produce the } \\
\text { copy of the vertex that is then sent away. }\end{array}$ \\
\hline prepareSendToWorker & $\begin{array}{l}\text { Plug-in point to transfer data from master to worker just before } \\
\text { the worker's traversal is invoked. }\end{array}$ \\
\hline prepareSendToMaster & $\begin{array}{l}\text { Plug-in point to transfer data from worker to master just before } \\
\text { the worker quits a traversal. }\end{array}$ \\
\hline mergeWi thWorker & Counterpart of prepareSendToWorker invoked on the worker. \\
\hline mergeWithMaster & Counterpart of prepareSendToMaster invoked on the master. \\
\hline prepareCopyToRemoteNode & $\begin{array}{l}\text { Event invoked just before data are migrated to another rank due } \\
\text { to dynamic load balancing. }\end{array}$ \\
\hline $\begin{array}{l}\text { mergeWi thRemoteDataDue- } \\
\text { ToForkOr Join }\end{array}$ & $\begin{array}{l}\text { Counterpart of prepareCopyToRemoteNode. Rebalancing } \\
\text { comprises two steps: a replicate of the tree parts is created on the } \\
\text { new remote worker and then the data are transferred through } \\
\text { these two events to the new worker. This way, data that can be } \\
\text { regenerated on-the-fly do not have to pass through the network. }\end{array}$ \\
\hline
\end{tabular}

Dynamic load balancing is hidden from the events. A distinct set of eventlike plugin points does exist. They allow the user to decide where to cut the tree, i.e., which subtrees to outsource to other ranks. It also allows to join two trees. Due to these routines, we may realize various load decomposition schemes. As out-of-the-box, proof-of-concept solution, Peano comes along with a greedy spacetree decomposition. It assumes that each tree node has the same computational cost. The critical path(s) within the global spacetree are identified, and the code then searches for the biggest subtree along this path that can be deployed to another rank. The algorithm continues until no further ranks are left or the tree is distributed completely.

\subsection{Communication Reduction and Elimination}

One showstopper in obtaining parallel scalability is any algorithmic synchronization (lock stepping). Synchronization in Peano materializes notably as vertical data exchange. To streamline the master-worker communication, we allow the global master to run a fixed number of grid traversals. All MPI ranks are then informed beforehand about this fixed number of traversals. At the same time, users may specify that local automata do not require information from coarser levels in these sweeps. Each rank thus runs a fixed number of iterations and couples to its multiscale neighbor, but the ranks are not globally synchronized with each other. We call one set of grid sweeps a batch.

The other way around, users can specify whether and which data are to be sent from workers to masters upon completion. This mechanism unfolds its full potential once we clarify that the wake-up call from a master decides whether vertical data transfer is required on a per rank per grid sweep base. Every time a worker traversal is started, the aligned event prepareSendToWorker on the master returns a flag, Peano memorizes this flag and, depending on it, skips the reduction 
from this worker. Users can explicitly disable inter-level (vertical) constraints from (1) between MPI ranks.

Besides vertical information, the code also offers routines to switch off horizontal data exchange via the stacks, users can minimize the horizontal data transfer through the heaps by sending out only those vertices/attributes that have changed, and we support algorithms that send out heap data in one iteration but receive them $n$ iterations later. This allows us to interweave grid traversals into a communication-demanding scheme: Data sent out in one iteration are allowed to run through the network while other grid traversals are executed.

Design Decision 13. Peano's default is a non-replicating scheme where all traversal automata are synchronized vertically, both top-down and bottom-up. Yet we allow the user codes to skip either synchronization. This decision can be made autonomously by each master per worker per grid traversal.

If all master-worker dataflow is masked out, then Peano's communication patterns resemble replicating schemes. All tree automata start to traverse, though with level shifts, at the same time. If all worker-master dataflow is eliminated, too, and global synchronization is realized by the user within the events manually through MPI calls, then the overall communication scheme is exactly the same as in a replicating strategy.

\subsection{Limitations of the Approach}

It remains open whether replicating or non-replicating spacetree decomposition are superior. Nonreplicating schemes tend to synchronize tighter, replicating schemes come at the cost of more data to be held consistent. They nevertheless seem to be more straightforward to program as synchronization does not block other ranks to continue their traversal. It thus might be reasonable, in the future, to offer both alternatives. Overlapping schemes fit into the presented mindset and have successfully been applied to spacetrees and SFCs. However, it is unclear, to the best of the author's knowledge, how the overlaps interplay with the dataflows of a scheme that remains nonreplicating wherever possible.

It is in this context important to observe that our distinction of bottom-up and top-down decomposition starts to blur depending on the usage: If solely data on the finest grid are held, then DFS in its unmodified form does not serialize the distributed tree traversal. While we can filter out grid events on coarser levels on a per-rank basis, i.e., for the non-distributed algorithm part, our code base also allows us to switch off reduction. This can be seen as parallel counterpart to the filtering. It makes the traversal pick up characteristics of leaf-only codes as detailed in the experiments. We finally remark that bottlenecks in reductions often are mitigated by the introduction of a tree structure and replication of coarse grid nodes.

\section{EXPERIMENTS}

We close our discussion with benchmarks to uncover some of the performance characteristics tied to the grid management, traversal, and programming paradigm. Our tests restrict to properties studied separately from each other. Real-world examples bringing together spacetrees with applications and thus also interweaving the individual properties are beyond scope. Examples for such configurations are discussed in the appendix or respective papers. Despite the lack of application context, we report runtime cost per grid entity-a quantity that has to change if an application plugs into Peano.

Throughout our experiments working with grids illustrated in Figure 5 we use six Peano instances holding 2, 11, 28, 768, 2,304 or 3,864 doubles per vertex. All floating point operations are removed, as they depend strongly on the particular application. Without arithmetic load, our setup 

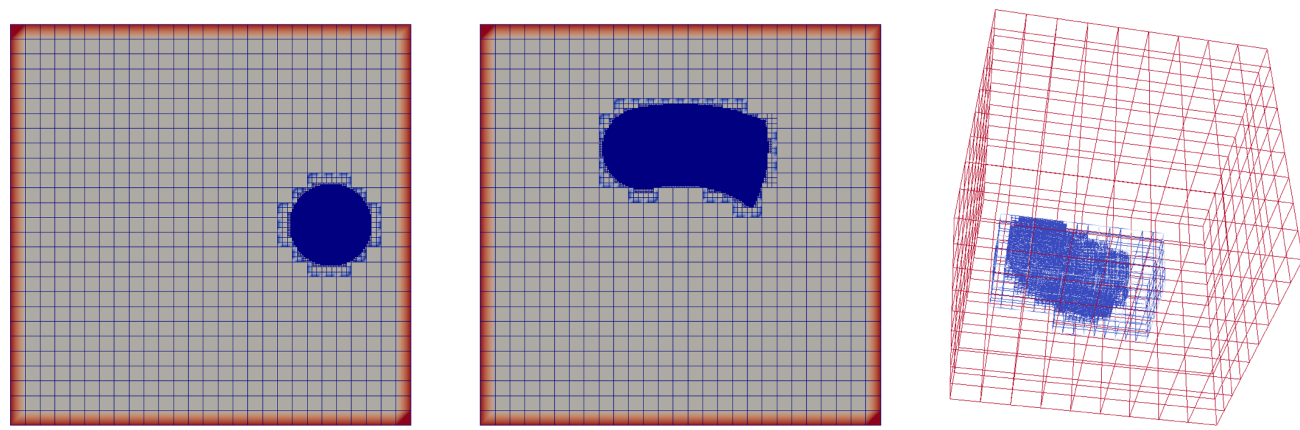

Fig. 5. Grid snapshots of our $d=2$ test setup at startup (left) and after few time steps (middle) with $h_{\max }=$ $3^{-4}$ and $h_{\min }=3^{-8}$, i.e., $\Delta \ell=4$. The circular region of refinement runs anticlockwise. Right: Latter situation for $d=3$ mesh with $h_{\max }=3^{-3}$ and $h_{\min }=3^{-6}$.

mirrors the Stream COPY benchmark (McCalpin 1995). Two unknowns per vertex have to be stored at least for any matrix-free equation system solve. They hold the right-hand side and the solution. If we store low-order discretization stencils per vertex, then the memory footprint grows to at least 11 doubles per vertex ( 9 for the stencil for $d=2$ plus the 2 unknowns) or 28 for $d=3$ if we have an analytic right-hand side. Patches within the spacetree can yield any memory footprint per grid entity, but 768 unknowns per cell or vertex, respectively, are a reasonable "small" count. It arises, for example, from the shallow water code (Weinzierl et al. 2014) with $16 \times 16$ patches and three unknowns (velocity plus water height). Lattice Boltzmann with D2Q9 using $16 \times 16$ subgrids at the same time yields 2,304 unknowns, while D3Q19 yields 3,864 (Neumann 2013).

Throughout the experiments, we manually prescribe the adaptivity: We first fix a minimum and a maximum grid resolution $\left(h_{\min } \leq h_{\max }\right)$. If they are equal, then we study a regular grid. Otherwise, we refine around a circle within the domain to the finest spacetree level meeting $h_{\text {min }}$ and coarse outside of this circle up to the coarsest mesh level meeting $h_{\text {max }}$. All data quantify the difference of $h_{\max }$ and $h_{\text {min }}$ as difference $\Delta \ell$ in levels. $\Delta \ell=0$ denotes the regular grid with mesh width $h_{\text {min }}$. No balancing (Isaac et al. 2012; Sampath et al. 2008; Sundar et al. 2008) is imposed. The "refinement" circle follows an ellipsoidal trajectory, i.e., the mesh adaptivity changes in each mesh sweep (Figure 5). The unit square or cube is our computational domain. If not stated otherwise, then we choose $h_{\max }=4.57 \cdot 10^{-4}$ for $d=2$ and $h_{\max }=4.12 \cdot 10^{-3}$ for $d=3$. The unit square or cube is the computational domain, and we start to refine from $h_{\max }$ onward.

We use Durham's Intel E5-2650V4 (Broadwell) cluster Hamilton with 24 cores per node. They run at $2.4 \mathrm{GHz}$ and are connected by Omnipath. Upscaling data stems from SuperMUC hosting Sandy Bridge-EP Xeon E5-2680 processors at 2.7GHz. All shared memory tests rely on Intel's Threading Building Blocks (TBB).

\subsection{Persistency Model and Data Management}

We start our experiments with single core measurements. Our deterministic, automaton-based grid traversal of a linearised spacetree pipes two data streams-one for the cells and one for the vertices-through the chip (Figure 2). Temporary, intermediate storage resides in the caches. Refinement or coarsening triggers stream insertions or the removal of elements from the output stream. This yields, despite the dynamic adaptivity, data accesses with high spatial and temporal locality (Kowarschik and Weiß 2003) and, hence, high cache hit rates (Bungartz et al. 2010b; Mehl et al. 2006; Weinzierl 2009; Weinzierl and Mehl 2011). Performance countermeasurements confirm this advantageous cache behavior for various application configurations (Table 6). 
Table 6. Performance Counter Data for a Two-dimensional Benchmark Setup

\begin{tabular}{c|rc|rc|rc|rc|rc}
\hline & \multicolumn{2}{|c}{2} & \multicolumn{2}{|c|}{11} & \multicolumn{2}{|c|}{768} & \multicolumn{2}{|c}{2304} & \multicolumn{2}{|c}{3864} \\
$\Delta \ell$ & \multicolumn{1}{|c}{ BW } & L2 & \multicolumn{1}{|c|}{ BW } & L2 & BW & L2 & \multicolumn{1}{c|}{ BW } & L2 & \multicolumn{1}{c}{ BW } & L2 \\
\hline 3 & 15.33 & 0.0004 & 28.67 & 0.0009 & $1,061.04$ & 0.030 & 863.16 & 0.06 & 839.41 & 0.12 \\
2 & 13.54 & 0.0005 & 12.70 & 0.0012 & $2,694.28$ & 0.037 & $2,020.64$ & 0.13 & $1,806.46$ & 0.45 \\
1 & 15.06 & 0.0004 & 27.07 & 0.0010 & $2,744.77$ & 0.037 & $1,999.11$ & 0.13 & $1,822.25$ & 0.48 \\
0 & 55.04 & 0.0006 & 174.40 & 0.0010 & $2,853.33$ & 0.036 & $1,365.75$ & 0.14 & $1,798.71$ & 0.48 \\
\hline 3 & 15.62 & 0.0008 & 31.78 & 0.0009 & $1,819.75$ & 0.010 & $3,327.44$ & 0.021 & $4,068.98$ & 0.027 \\
2 & 7.32 & 0.0009 & 10.74 & 0.0010 & $3,941.51$ & 0.013 & $7,426.19$ & 0.030 & $8,951.68$ & 0.041 \\
1 & 48.29 & 0.0009 & 47.10 & 0.0010 & $4,156.80$ & 0.013 & $7,556.84$ & 0.030 & $9,179.52$ & 0.041 \\
0 & 140.22 & 0.0009 & 215.27 & 0.0010 & $4,404.97$ & 0.014 & $7,822.45$ & 0.030 & $7,833.64$ & 0.041 \\
\hline
\end{tabular}

We compare stack- (top) to heap-based (bottom) data management. Bandwidth is given as MB/s while L2 denotes the L2 cache miss rate. $\Delta \ell=3$ uses around 45,000 spacetree vertices per step, while the regular grid $(\Delta \ell=0)$ reads and writes the data of 110,000 vertices.
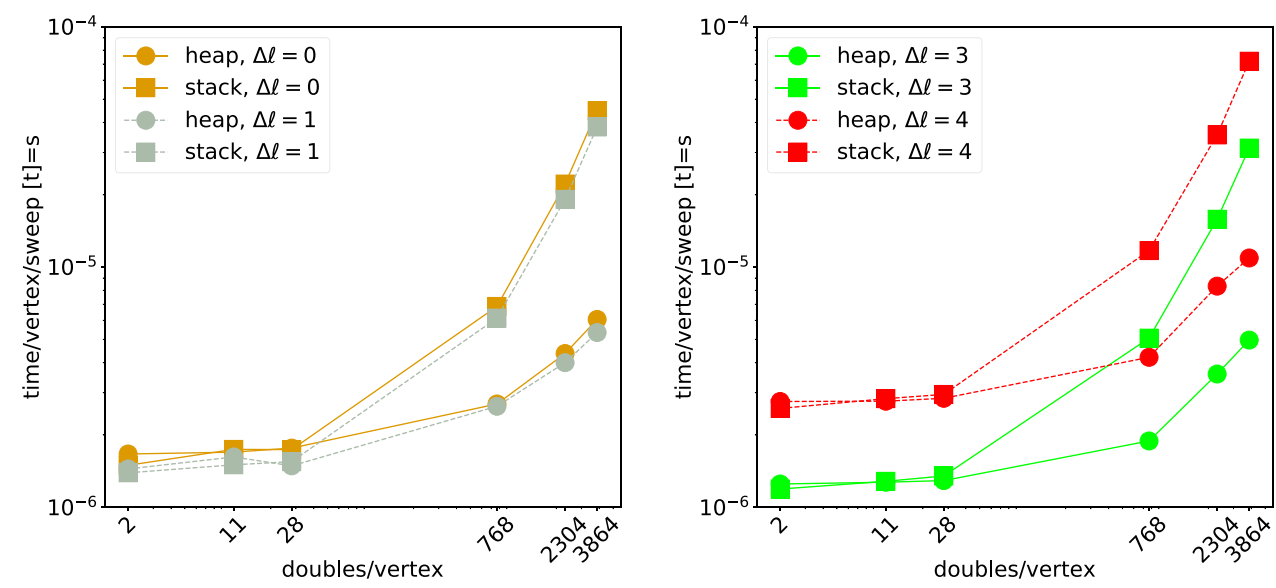

Fig. 6. Time per vertex per grid sweep for different level differences and dynamically adaptive $2 d$ meshes. All floating point operations disabled.

Our single core measurements tracking runtimes (Figure 6) compare stack- to heap-based unknown storage. For the setups with a small persistent memory footprint per grid entity, our approach is spacetree administration bound, as the runtime profile is independent of the data stored per grid entity. From 28 doubles per vertex onward, the runtime increases with increasing data cardinality. The code's administration overhead team up with data squeezing through the memory interconnect and caches. This holds for both storage paradigms.

OBSERVATION 6. In the plain algorithm formulation, the administrative cost per vertex is almost agnostic of the refinement pattern.

Even in the "worst case" where (i) the mesh is extremely adaptive, (ii) changes in each and every time step, and (iii) each vertex has very small memory footprint, the AMR overhead is not more than a factor of two. However, memory allocations do slow down the code once the memory footprint per vertex becomes reasonably high.

Deploying user data to the heap yields a higher effective memory throughput than sole stackbased storage. It also yields a better cache hit rate. As the heaps implicitly exploit the SFC/DFS locality through the tree access keys, these observations imply that the stack-based approach 
suffers from non-compulsory misses as data are written forth and back to temporary stack containers. Yet the heap-based approach introduces one further memory access indirection that renders a stack-based approach superior for very small memory footprints (2 or 11 doubles per vertex only). Embedding application-specific data into the spacetree stream is advantageous if and only if the number of doubles per vertex is small. As soon as we store more than a few doubles, it is advantageous to separate the spacetree stream from the actual user data. For large data cardinalities, we otherwise quickly lose up to an order of magnitude of performance. All observations also hold qualitatively for $d=3$.

OBSERVATION 7. For very small data cardinalities per grid entity and rapidly changing grids, it pays to merge the compute data into the grid data. Otherwise, it is better to separate the two containers.

These data confirm that effective grid implementations have to exploit any grid regularity or time invariance to bring administrative overhead down. The other way around, they suggest that effective codes based upon trees should exploit and introduce data regularity. Examples for this are block-structured or Structured AMR (SAMR) approaches (Deiterding 2005; Dubey et al. 2016; Schornbaum and Rüde 2018; Weinzierl et al. 2014) that strive for hybrids of free adaptivity and data regularity. Overall, a code that is solely tree based remains significantly below the throughput threshold of the Stream COPY benchmark (McCalpin 1995) on a single core. For the latter, we obtain $18,000-19,000 \mathrm{MB} / \mathrm{s}$ for setups that pick up memory footprints from the $\geq 768$ doubles-per-vertex configurations. With smaller per-vertex footprint, Stream COPY throughput becomes worse-down to $13,000 \mathrm{MB} / \mathrm{s}-$ until we reach a situation where the data footprint fits into a cache. This holds for all memory footprints corresponding to 2 double-per-vertex and the adaptive 11 double-per-vertex choices. While Stream starts to exhibit throughputs significantly above $20,000 \mathrm{MB} / \mathrm{s}$ then, the tree traversal's administrative overhead stops us from benefitting from the fits into cache. For such tiny setups, a multiscale set of regular Cartesian meshes (arrays) is superior to the AMR tree idea.

\subsection{Transformation of DFS into BFS}

Despite good memory access characteristics, stream-based data management suffers from data movements. The pressure on the caches is high as data are permanently written and read from and to the caches. Notably, vertices are written forth and back up to $2^{d}$ times. The more user data we hold on heaps, i.e., the fewer data are embedded into the tree stream, the smaller the impact of this overhead. Still, the whole tree administration is not negligible. We therefore propose in Section 5.2 to cut out regular subgrids from the global grid. If all user data are stored on heaps, i.e., no data are embedded into the (linearized) stream, then no user data are unnecessarily moved by the grid traversal anymore within the regular subtrees.

Runs on the Broadwell (Figure 7) compare a plain realization to a realization exploiting the grid regularity through an on-the-fly switch into BFS. This realization also stores regular subtrees separately. If a part of the spacetree is stationary and regular, then the traversal requires one kickoff grid sweep where (2) is determined. If $f \geq 0$, then the next iteration switches locally to BFS. If a grid segment remains stationary and regular after the first BFS sweep, then it is cut from the global grid and stored separately. The three-step pattern is clearly observed for the regular grid. We note that the very first grid sweep is the grid construction where $f=\perp$ everywhere by definition. Besides the three-step pattern, runtime peaks are caused by memory allocations for the regular subtrees and whenever regular subtrees cut out of the mesh are reintegrated into this very one due to dynamic mesh refinement or coarsening.

An evaluation of (2) and an on-the-fly switch to BFS for regular subtrees speeds up traversals by up to a factor of 10. For the regular subgrids, it is advantageous to embed the user data into the tree. 

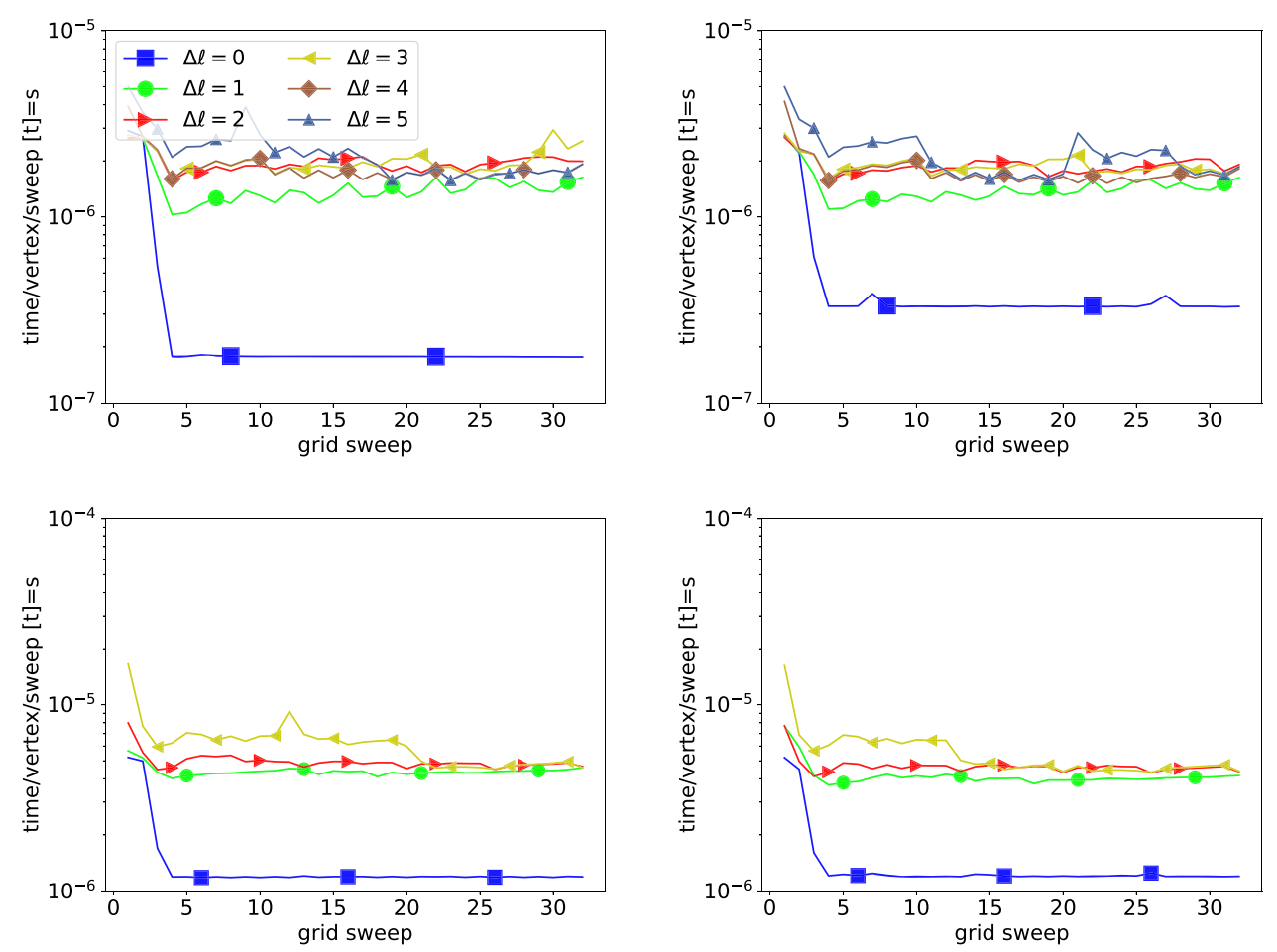

Fig. 7. Cost (real time) per grid sweep per vertex on a Hamilton node. $d=2$ (top) vs. $d=3$ (bottom) with 28 doubles per vertex. We compare stack-based user data management (left) to heap-based storage (right). All floating point operations eliminated. $\Delta \ell$ denotes the AMR levels, so $\Delta \ell=0$ identifies a regular grid.

This is a surprise as heap-based storage for regular subgrids means that the grid data are solely read but no data are written. Heap-based storage. however. introduces scattering. The regular grid levels stored as continuous block link to scattered data on the heap. Unaltered grid regions are key to improve performance. For them, there is an overhead through the traversal reordering that is compensated by the simplified event invocation and the elimination of case distinctions.

OBSERVATION 8. On-the-fly tracking of regular subtrees pays off. Replacing regular grid regions within the tree is advantageous if the grid does not change often, if the regular subtrees held separately are sufficiently large, and if few doubles are held per grid vertex.

Cutting out regular subgrids from the overall grid is delicate as we have to keep the redundant vertices between the grid regions consistent. This administrative overhead, which arises mainly for the stack-based storage, is only amortized if the persistent subregions are large $\Delta \ell \in\{0,1\}$. Our observations suggest that it is, in practice, unavoidable to use some kind of patches or high-order methods with lots of unknowns per grid cell if high FLOP rates are to be obtained. With the full multiscale tree formalism, we need a significant workload to mitigate the spacetree administration overhead. This notably holds for $d \geq 3$ where huge regular subgrids are less likely to arise.

\subsection{Concurrency Impact of the DFS-BFS Transformation}

We continue with multicore experiments handling solely the finest grid level-we label those with finegrid-or traversing all grid levels (label multiscale). The access patterns simulate stencil (matrix-free operator) evaluations. The latter-mirroring data accesses of an additive multigrid 

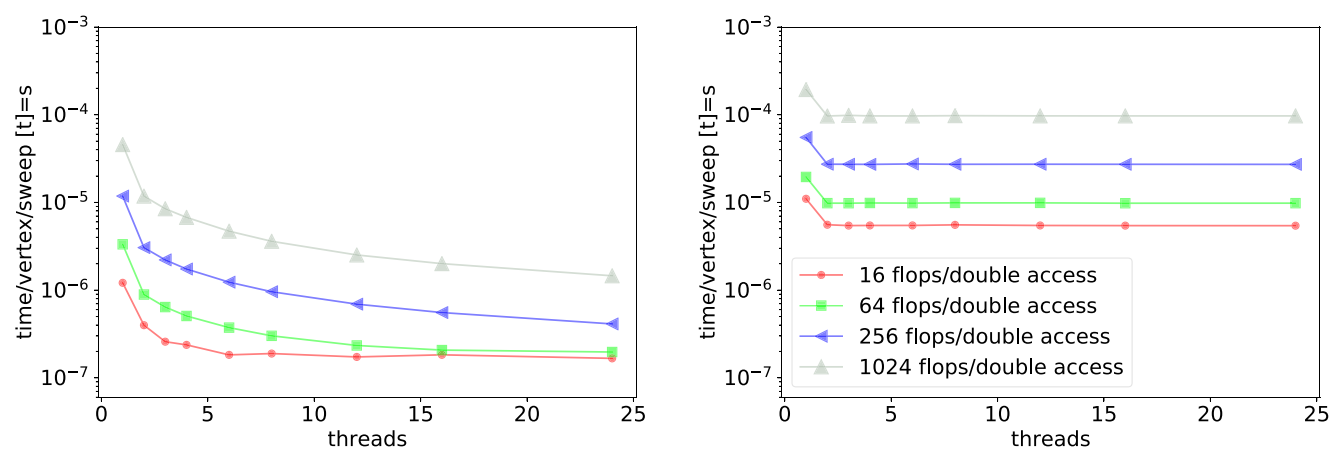

Fig. 8. Scaling curves on Hamilton for $d=2$ setups with different arithmetic load per vertex. Two doubles are held per vertex, and we process all levels (multiscale). Regular grid (left) vs. dynamically adaptive grid (right) with $\Delta \ell=2$.

scheme-avoids race conditions between multiscale vertex accesses through proper mesh colouring with $7^{d}$ colours, while the finegrid variant succeeds with four colours in total. Again, we run the setups for various combinations of maximum and minimum mesh sizes. All experiments not employing regular grids work with dynamic adaptivity changing each grid sweep.

Various shared memory parallelization features of Peano's traversal automaton can be switched on or off by the user code, and its traversal automaton using static problem partitioning for all parallel loops can also be fed with situation-specific grain sizes. We thus face a large range of parameter choices: Which features are to be switched on and off and which grain sizes are to be chosen? For the present manuscript, we use Peano's hard-coded default values.

Its outcome can qualitatively be summarized as follows: Significant scalability is obtained for the colouring of the BFS traversal phases where we loop over the regular subtrees' levels with a parallel for. The more computational load per grid entity the better the scaling. The parallel efficiency is lost as soon as we run into dynamically adaptive grids (Figure 8). Only if large regular sub-grids are encountered do we observe scaling. Variants with only finegrid manipulations scale better if the work per vertex is sufficiently high-an obvious property given the weaker concurrency constraints. Furthermore, we observe scaling for adaptive subpatterns if and only if the adaptivity pattern remains stationary The last two properties are not shown here. Decomposing the data load and store process along the surface of regular subtrees into tasks is robustly superior to a sequential load and store. Once we load data for $f \geq 1$, we may furthermore hide the handling of coarse levels behind the load and stores of finer levels. Both techniques (Schreiber et al. 2013b) yield a parallel speedup of two. The effect is visible best for the dynamically adaptive grid that otherwise does not scale at all, i.e., it benefits solely from the parallelised loads/stores and the overlapping of processing and memory accesses.

Finally, we remark that a proper grain size should scale with the problem size: If the grain size is too small relative to the problem size, then our academic decomposition into tiny tasks yields a too-high task administration overhead. Peano offers a plug-in point to inject proper problem size dependent into any application. If a manual identification is too cumbersome, then its toolbox collection provides a generic machine-learning algorithm to derive proper grain size choices onthe-fly (Charrier and Weinzierl 2017).

Strong dynamic adaptivity makes our DFS/BFS-based parallelization deteriorate from a parallelization strategy into a minor speed improvement. This teaches five lessons: First, we have to employ classic domain decomposition working with separated trees to obtain good scalability in our AMR context. This allows threads to work on separate memory regions and can be done ei- 

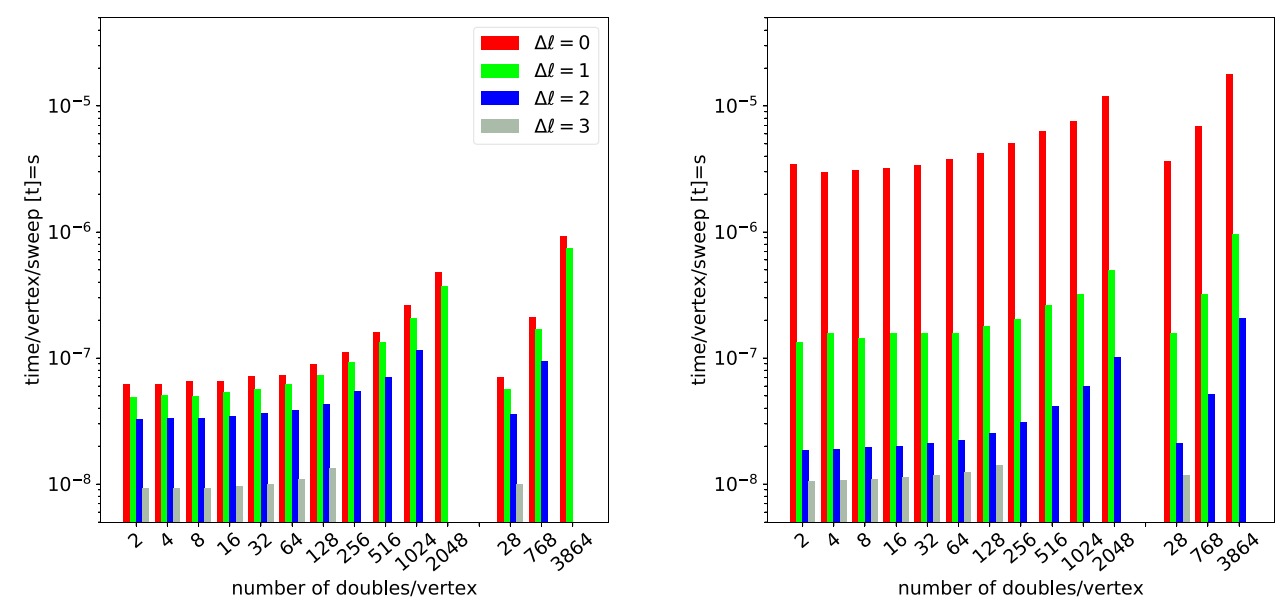

Fig. 9. Average runtime per grid sweep on SuperMUC for $d=2$ (left; 10 ranks) and $d=3$ (right; 28 ranks) against memory footprint per vertex. The three measurements to the right in each graph $(28,768,3,864)$ pick up typical parameter choices from other experiments from the paper.

ther via MPI as studied next or with low-overhead shared memory (compare Schreiber et al. (2013a, 2013b), e.g.). Data decomposition has to be applied on-node, too. Second, scaling a Peano code is simplified if the basic operations per grid entity themselves exploit multiple cores (compare to the concepts of inter-patch and intra-patch concurrency in Weinzierl et al. (2014)). A pure gridbased parallelization easily falls short of exploiting all cores. Third, it makes sense to (artificially) increase the grid regularity. The loss in efficiency measured by work per accuracy can be compensated by an increased scalability. Fourth, it makes sense in a multicore environment to fix the grid over multiple grid sweeps. Through temporal blocking, we can eliminate thread synchronization. Finally, it is reasonable to evaluate where tasks can decouple from the grid traversal.

Splitting of tree processing, i.e., working in a logically distributed environment, is next discussed in a (logically) distributed memory context. Decoupling of grid traversal and tasking naturally leads to a producer-consumer pattern: The grid traversal then creates tasks that are processed by other threads. In Eckhardt et al. (2015), we use such a mechanism to deploy data conversions after a cell processing to background threads. The workstream pattern from Turcksin et al. (2016) establishes a similar dataflow. In such a producer pattern, the (limited) scalability of the mesh traversal becomes advantageous. The little bit of scaling ensures that tasks are created reasonably quickly, while the limited concurrency ensures that the node does not hinder all cores to process actual work. Again, it becomes obvious that for very large regular (sub)trees, it is convenient to disable such dataflow patterns and instead fall back to direct DFS-BFS transformation.

\subsection{Distributed Memory Scalability}

Our tree decomposition experiments enable the BFS/DFS transformations but switch off any shared memory feature. The tree decomposition is realized with MPI. We start with a setup with 10 ranks $(d=2)$ or 28 ranks $(d=3)$, where we configure the code to hold different number of unknowns per vertex. Though we remove all computations from the code, it does exchange all data along the domain boundary. This yields a communication worst-case setup.

Qualitatively similar to the serial data, we see an almost-constant runtime per sweep (Figure 9) as long as we hold up to 64 doubles per grid entity. Once we go beyond 64 doubles per vertex, the 
runtime starts to depend on the data held per vertex. It slowly grows into a linear relation. For $d=3$, we obtain comparable behavior.

For small relative memory footprints, our code is spacetree administration bound; it is not bandwidth influenced, neither with respect to memory bandwidth nor with respect to network bandwidth. All data exchange can hide behind the traversal because of the discussed SFC stream paradigm that allows the automaton to send out data while they still run through the grid. For large memory footprints, our code cannot hide data transfer behind the traversals anymore. The fact that the time-to-memory footprint relation is transitioning into a linear relation slowly shows that the code continues to succeed in hiding some data exchange behind the actual grid traversal. However, the more data there are to be transferred the less significant this hiding.

OBSERVATION 9. The deterministic, automaton-based traversal allows the automaton to hide data exchange automatically behind the user-defined events. This is behavior known for codes that work solely on $\Omega_{h}$. Our data reveal that we can recover the characteristics for multiscale data.

We continue with classic upscaling and launch a sequence of $n$ grid sweeps. Then we run the same simulation again but trigger always two grid traversals as one batch. Then, we do four sweeps in one batch, and so forth. Each experiment, i.e., $n$-configuration, is done four times: We either preserve all vertical data exchange, or we switch off the wake-up from master to worker within one batch-the worker-master synchronization then results from the exchange of the multiscale domain boundary data-skip worker-master reductions or eliminate both; all elimination is only done between batches. As soon as a batch of grid sweeps terminates, we restrict all data vertically. When a batch is kicked off, we run all master-worker data sends.

Our measurements reveal strong scaling effects in combination with an amortization of administrative overhead (Figure 10): All timings stagnate the later and the higher the vertex count. Furthermore, the higher the vertex count the lower the cost per vertex for a fixed rank number. Grouping grid sweeps into batches alone does not pay off. Minimizing horizontal data exchange, i.e., to exchange only vertices that are updated, improves the performance slightly. Both techniques combined yield fast code once we also skip vertical data exchange within a batch. If we compare an elimination of worker-master to worker-master information exchange, then a skip of the reductions contributes more to good scalability. This diversification with respect to vertical data exchange becomes observable when the graph enters the strong scaling stagnation regime. For several setups, it can invert the classic strong scaling behavior, i.e., the expectation that a simulation scales the better the more detailed the grid that is used is. This behavior is reasonable once we emphasize that finer grids couple individual ranks stronger through horizontal data exchange than shallow grids.

OBSERVATION 10. The elimination of vertical data exchange in combination with batching allows us to decouple ranks that do not exchange a significant amount of data through the domain boundaries.

The advantageous behavior with the data exchange skips does not eliminate the fact that there are massive steps in the scalability graphs: Our naïve, static dummy load balancing cuts through the spacetree in a top-down fashion: Optimal scalability thus is obtained if and only if the number of ranks matches the grid structure. Notably, whenever the mesh is very close to regular while the rank count is from $\left\{k^{i d} \mid i \in \mathrm{N}^{+}\right\}$, this approach yields perfect partitions. These are the best cases in the graph. For a $d=2$ regular grid, a rank count of nine, for example, allows us to split up a regular grid properly. If only two ranks are available only, then a proper splitting would exhibit an ill balancing of 4:5. The master-worker topology, however, permits only ratios of 1:8. The effect becomes stronger for $d=3$ (not shown) and explains the plateau at 4.096 ranks. 

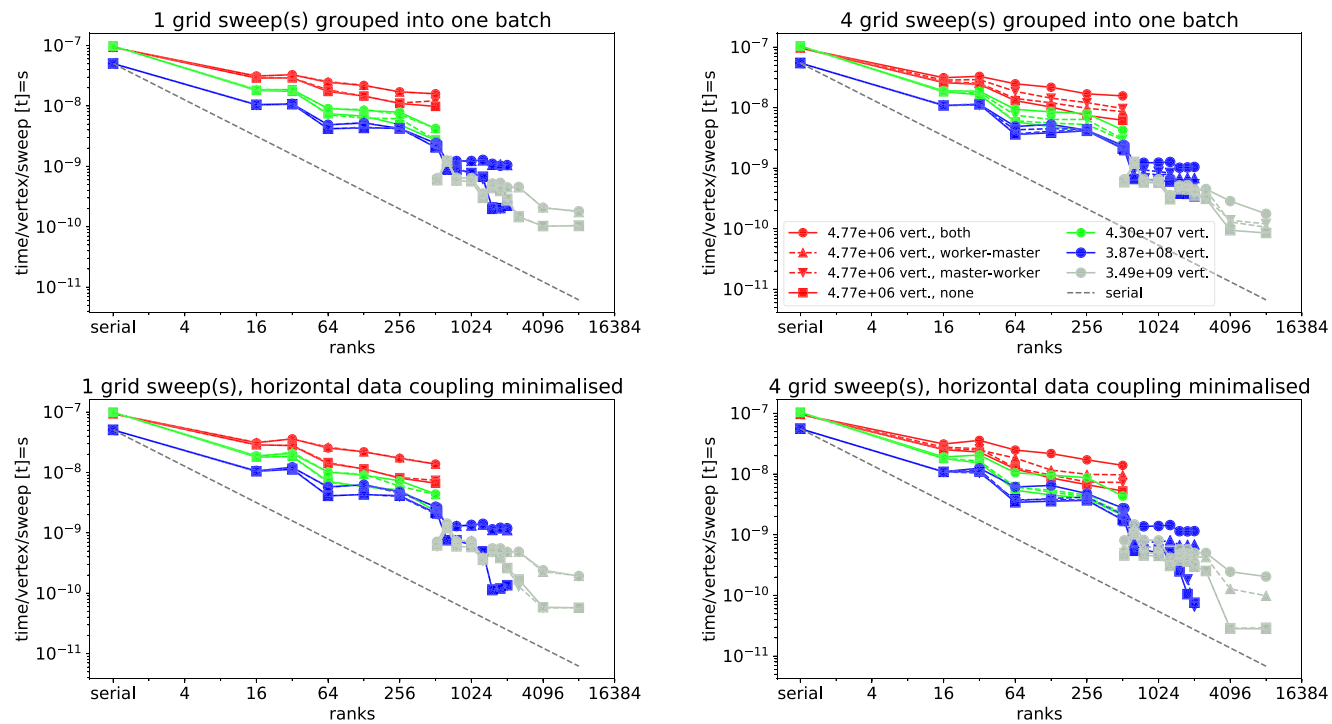

Fig. 10. $d=2$ scalability as runtime per sweep per vertex on SuperMUC. Eight doubles are assigned to each vertex. In the top row, each and every vertex along the domain boundary is exchanged with all neighboring partitions. In the middle and lower rows, vertices are only exchanged when they change their state. We either switch on vertical communication both ways, send only data from the masters to the workers, the other way around, or eliminate all vertical data exchange per batch completely, i.e., exchange vertical data only at the end of the batch. No arithmetic work is done.

OBSERVATION 11. The master-worker MPI topology restricts the admissible finegrid partitions. Many reasonable finegrid splittings cannot be mapped onto a master-worker topology and can only be approximated. While the non-replicating scheme minimizes the data and work done per rank, it struggles to compete with replicating schemes.

There are two straightforward solutions to this challenge. We either can combine a non-replicating scheme on coarser resolutions with a replicating domain decomposition for finer scales. This is subject of future work-besides the development of better non-greedy load balancing schemes that approximate the best-case partitionings better. The latter is a subject independent of Peano as it is connected through a well-defined, small API. Complementary, we can deploy multiple MPI ranks per compute node, derive a proper domain splitting-in Peano's case using the Peano SFC is a natural candidate-and ensure that workers responsible for subdomains neighboring along the SFC are deployed to the same rank. This technique smoothes out the scalability to some degree (Table 7) once the ratio data per vertex relative to the mesh size becomes reasonably small. It seems to be particularly promising for applications that cannot exploit all cores through shared memory parallelization. It seems not to be promising for $d=3$ setups with massive data per vertex and, thus, massive communication demands. We, however, emphasize that our results in Table 7 are biased as we strip the code off any computation.

Cutting a tree into distributed memory pieces has the advantage that it suits both distributed and shared memory machines. We close our distributed tree discussion by running the tree decomposition on a manycore architecture as alternative to our DFS/BFS transformation. The latter is orthogonal, i.e., it can be combined with the decomposition. Our results (Figure 11) suggest that such an on-node strategy can yield another speedup of close to a factor of almost 20 with high efficiency. Going beyond a factor of 20 or overloading do not pay off. We notably should refrain 
Table 7. Some Speedups for Various (Averaged) Vertex Counts in $\Omega_{h}$ Where We Use the Peano SFC to Derive Proper Domain Decompositions and Deploy Six Ranks per 24 Core Compute Node

\begin{tabular}{|c|c|c|c|c|c|c|c|c|}
\hline \multirow{2}{*}{$\begin{array}{l}\text { nodes/ } \\
\text { ranks }\end{array}$} & \multicolumn{4}{|c|}{$d=2$} & \multicolumn{4}{|c|}{$d=3$} \\
\hline & $5.90 \cdot 10^{4}$ & $5.31 \cdot 10^{5}$ & $4.78 \cdot 10^{6}$ & $4.30 \cdot 10^{7}$ & $5.31 \cdot 10^{5}$ & $1.43 \cdot 10^{7}$ & $3.87 \cdot 10^{8}$ & $1.05 \cdot 10^{10}$ \\
\hline$\overline{1 / 6}$ & 2.89 & 4.39 & & & 2.13 & 3.99 & & \\
\hline $2 / 12$ & 10.1 & 13.4 & & & 3.04 & 4.79 & & \\
\hline $4 / 24$ & 10.4 & 12.4 & 20.9 & & 3.07 & 13.7 & 17.0 & \\
\hline $8 / 48$ & 10.7 & 21.2 & 32.2 & & 3.05 & 9.35 & 23.6 & \\
\hline $12 / 72$ & 11.3 & 24.2 & 34.4 & & 3.05 & 9.28 & 24.5 & \\
\hline $16 / 96$ & & 29.6 & 85.2 & & & 9.26 & 24.6 & 79.7 \\
\hline$\overline{1 / 6}$ & 1.59 & 2.48 & 3.52 & & 0.84 & 2.13 & 4.05 & \\
\hline $2 / 12$ & 1.35 & 8.74 & 12.7 & & 0.78 & 3.03 & 4.84 & \\
\hline $4 / 24$ & 2.72 & 8.39 & 11.0 & 19.4 & 0.77 & 3.05 & 14.0 & 17.2 \\
\hline $8 / 48$ & 3.00 & 8.85 & 17.9 & 28.1 & & 3.04 & 9.38 & 23.9 \\
\hline $12 / 72$ & & 8.97 & 19.5 & 30.7 & & & & \\
\hline $16 / 96$ & & & 40.0 & 86.9 & & & & \\
\hline
\end{tabular}

The speedups are normalized to the smallest problem setup. We present data for 2 (top) and 768 (bottom) doubles per vertex. All computation is stripped off from the code.
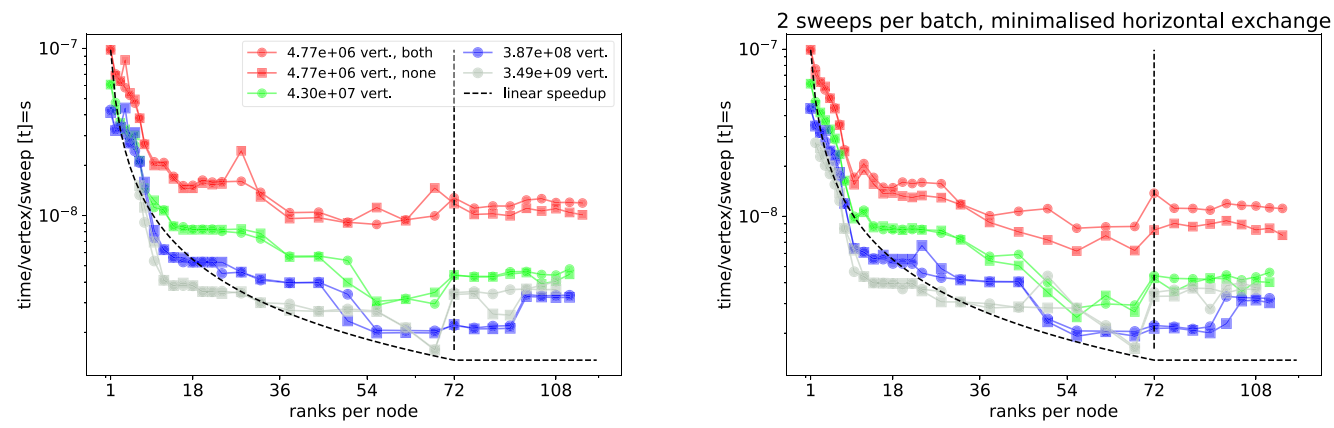

Fig. 11. Cost as time per sweep per vertex for $d=2$ on one KNL node with 72 cores. The spacetree is decomposed into subtrees and distributed via MPI. No BFS parallelization is enabled.

from booking all cores for the computation. Vertical data exchange or its skipping, respectively, do not play a major role for larger trees. They unleash their power between the nodes.

ObSERVATION 12. A mixture of tree decomposition with on-the-fly BFS/DFS transformations is a promising strategy to exploit manycore architectures.

We conclude that Peano's "vanilla" grid traversal has upscaling potential, though it is limited. Yet all presented setups are worst case: They change the grid in each and every grid sweep, they process all resolution levels, and they couple these levels with each other. Notably, they only move data through the system but do not compute. Under these constraints, even the combination of space-filling curves with traversal optimisation techniques does not guarantee for scalability-despite the fact that data exchange can be hidden behind the traversal to are large extent.

The limited scalability does not allow us to draw conclusions about the well-suitedness of trees and SFCs for parallel computing in general or on the potential of Peano; notably as many publications rely successfully on the SFC ingredients (Bader 2013; Bangerth et al. 2011; Bungartz et al. 
2006; Burstedde et al. 2011; Deiterding 2005; Dreher and Grauer 2005; Griebel and Zumbusch 1999; Meister et al. 2012; Rahimian et al. 2010; Sampath et al. 2008; Schornbaum and Rüde 2018; Schreiber et al. 2013a, 2013b). It allows to draw a conclusion on their application: Scaling a code requires us to weaken one or several of the constraints, and we have introduced significant computation.

It seems to be necessary that either very high computational load per grid entity is applied or the grid acts as metadata structure hosting reasonable large subproblems that scale internally. Examples for such applications are very high order approaches alike (Charrier and Weinzierl 2018) or hybrid approaches as we find them in Bergen and Hülsemann (2004) and Weinzierl et al. (2014). Here, regular subgrids are embedded into the actual (spacetree) meta mesh. Orthogonally, it is recommendable for scaling codes that we do not couple all resolution levels in each and every sweep or that we keep the grid stationary over multiple grid sweeps. We then can exploit temporary and spatial grid regularity.

\section{CONCLUSION AND OUTLOOK}

The present article introduces a software framework for dynamically adaptive multiscale grids that fuse data management and data traversal as well as, if not explicitly outsourced to a heap, application data storage. The underlying programming model is formalized with two automata yielding an event-based algorithm development approach. Besides the fact that the software delivers a merger of multiple state-of-the-art features such as support for arbitrary dimensions, multiscale data representation, and low memory footprint capabilities, it is resonably simple to handle due to its restrictive, minimalistic programming model. This makes the software well suited for fast prototyping as well as bigger codes with a clear separation of concerns as long as they accept the restrictive academic programming paradigm that might imply that not each legacy code can directly use the AMR features.

The manuscript characterizes, classifies, and motivates several design decisions made while the framework was written. It also explicitly highlights shortcomings and open questions notably in comparison with other approaches found in literature. The references to alternative pathways toward realization are backed up with some experiments that highlight that the software is capable to code sophisticated applications running in parallel. Yet the discussion and the results also highlight that other spacetree code developers might favour other realization variants that are superior for particular challenges.

There are two natural directions of future work. Both start from the observation that the present discussion is very academic and computer science-centric. It lacks challenges from realworld computational simulation codes that rely on the present software stack, some of its components, or discussed paradigms. On the one hand, it is important to track their code maturity, maintainability, and clarity once we "scale" up the code with respect to simulation detail complexity. This will allow us to assess and understand the limitations and drawbacks imposed by the present programming model from a usability point of view. It also might help to identify ways to make the software easier accessible without giving up its clarity and clear separation of concerns.

On the other hand, it is important to track the computational and algorithmical efficiency of application codes. Our case studies reveal that there is scalability potential arising from the present code base. Yet this scalability, though we restrict to worst-case studies that will not be found this way in applications, is far from optimal and will drive future developments. We see notably potential in mergers of replicating and non-replicating tree decomposition schemes and in the combination of our grid-based task model with tasking approaches where tasks are decoupled from the grid entities and can run in parallel to the grid traversal. 


\section{APPENDICES}

\section{A SOFTWARE BASE}

Peano is freely available from (Weinzierl et al. 2015). We offer both tarballs and repository access through Subversion. Support is provided through a gitlab discussion board. An extensive guidebook discusses how to implement solvers in Peano. It also comprises case studies.

The code baseline offers support for adaptive Cartesian grids (Akcelik et al. 2003; Bangerth et al. 2007; Burstedde et al. 2011; Gadeschi et al. 2015; Griebel and Zumbusch 1999; Jeong et al. 2001; Khokhlov 1998; Kolobov and Arslanbekov 2016; Lashuk et al. 2012; Robey et al. 2013; Sampath et al. 2008; Sundar et al. 2012, 2008; Teunissen and Ebert 2017; Tumblin et al. 2015) as discussed in the manuscript. It thus follows an academic approach to AMR programming and lacks the flexibility and generality of software alike Adams et al. (2016b), Bangerth et al. (2007, 2016), Bastian et al. (2008a, 2008b), and de St. Germain et al. (2000).

Extensions of the sole grid and its traversal are available via a template mechanism and small routine collections (toolkits) that inject plotting facilities for Paraview/VisIt, realize shared memory autotuning (Charrier and Weinzierl 2017) or add dynamic load balancing based upon graph partitioning or the underlying Peano space-filling curve. Further examples for toolkits are routine collections for matrix-free multigrid (Reps and Weinzierl 2017; Weinzierl and Weinzierl 2018), Particle-in-Cell features (Weinzierl et al. 2016) similar to (Kolobov and Arslanbekov 2016) or patchbased PDE solvers (Weinzierl et al. 2014). The latter allows users to compose the AMR grid as assembly of regular patches and makes the software resemble block-structured AMR alike Adams et al. (2016b), de St. Germain et al. (2000), e.g.

The software currently offers bindings to TBB for task-based parallelization. These bindings are hidden through a software layer. With task approaches having successfully been used in various spacetree and non-spacetree codes such as (de St. Germain et al. 2000; Meister et al. 2012; Weinbub et al. 2014), the bindings could be replaced by a more sophisticated task subsystems.

Peano supports native $\mathrm{C}$ user data natively. If users want to model more complex data structures per cell or vertex, respectively, then Peano relies on DaStGen (Bungartz et al. 2008, 2010a). DaStGen is a $\mathrm{C}++$ augmentation that automatically equips $\mathrm{C}++$ classes with MPI data types, data compression, different class variants distinguishing temporary from persistent object attributes, and so forth. Peano's internal data structures all are modelled with DaStGen. If users rely on stack data management, then the (maximum) data cardinality per vertex or cell, respectively, has to be known at compile time. If users rely on heaps, then the number of objects per vertex or cell-either native $\mathrm{C}$ types or DaStGen objects-is flexible, i.e., may grow and shrink.

\section{B REMARKS ON SOME PEANO APPLICATIONS}

In Section 3.1, we sketch which types of applications do fit to our restrictive API. The present section substantiates these statements with examples from recent projects and further remarks.

Tripartitioning. From an application's point of view bi- and tripartitioning both come along with pros and cons. Aggressive $k=3$ coarsening can pose a challenge to geometric multigrid algorithms, e.g., if a too large spectrum of solution modes is thrown away per coarsening step. In return, finegrid cell centres coincide with coarse ones for tripartitioning. This simplifies the coding of cell-centred discretizations. Another pro/con pair is the transition from one resolution $h$ into another resolution $\hat{h}>h$ if 2:1-balancing is enforced: Our tri-partitioning creates a denser communication/data-exchange pattern than bipartitioning. In return, very fine resolutions $h$ do not spread out that significantly into coarser regions with $\hat{h}$. Different considerations might arise for different applications. 
Low-order, d-linear Finite Element Examples. In (Reps and Weinzierl 2017; Weinzierl and Weinzierl 2018), we study variants of geometric-algebraic multigrid solvers for Helmholtz and convection-diffusion equations. The Helmholtz setup studies high-dimensional grids with complex-valued grid spacing, while the convection-diffusion studies derive mergers of algebraic and geometric multigrid algorithms that combine the geometric efficiency and memory modesty with the algebraic robustness. Both articles introduce single-touch algorithms and both study loworder discretizations, i.e., compact 9-point stencils $(d=2), 27$-point stencils $(d=3), \ldots$ They solve the PDEs

$$
\begin{aligned}
-\Delta u+k^{2} u & =b \quad \text { and } \\
-\nabla(\epsilon \nabla) u+v \cdot u & =b
\end{aligned}
$$

with $u \approx \sum_{i} u_{i} \varphi_{i}$ through a Ritz-Galerkin formulation where $\varphi_{i}$ is a $d$-linear shape function centred around a non-hanging vertex on $\Omega_{h, \ell}$ and spanning $2^{d}$ cells.

Both articles use full approximation storage (FAS), i.e., they store a real representation of the solution on each grid level. They work on generating systems. This renders the treatment of hanging vertices straightforward. It exploits the fact that Peano holds the individual grids $\Omega_{h, \ell}$ separately, i.e., each vertex is unique through its level plus position and multiple vertices coinciding spatially can hold different data: finegrid vertices membering $\Omega_{h}$ encode the solution while coarser vertices at the same position hold FAS data plus multigrid correction terms. As all stencils are compact, they decompose additively over the $2^{d}$ adjacent cells and thus can be evaluated element-wise.

We augment each vertex by a residual holding a vertex's $r=b-A u$ contribution if $A$ is the system matrix arising from (3). Our application automaton plugs into touchVertexFirstTime to set $r \leftarrow 0$. Within enterCell, it reads in the $2^{d} r, u$ and $b$ values and contributes the residual contributions from the respective cells. The residual is accumulated. When the traversal automaton triggers touchVertexLastTime, we know that the residual for one vertex is accumulated completely, and we can trigger a smoothing step or residual restrictions. As Peano offers the opportunity to plug into the traversal automaton's steps up and down in the tree, inter-grid transfer operators can be realized "element-wise", too. Traversing the whole spacetree in one rush mirrors the behaviour of additive multigrid schemes. If we run through the levels sweep by sweep, i.e., mask out some levels, then the traversal mirrors the data access of multiplicative multigrid.

The Helmholtz setup stores all quantities directly within the vertices, i.e., it fuses spacetree grid data and solution values. Each vertex holds one double being the weight $u_{i}$ within (3) plus the discretised right-hand side. Additional temporary variables holds the residual, hierarchical residuals, error estimators, and so forth. The convection-diffusion code starts from this data representation, too. Yet it also stores inter-grid transfer stencils and discretization stencils, and those quantities are held on Peano's heap as they consist of at least $2 \cdot 5^{d}+3^{d}$ doubles and we furthermore study techniques how to convert them on-the-fly into non-IEEE representations where the resulting total memory footprint is not known a priori. With the work meandering around additive multigrid and BPX as preconditioner, Peano's DFS approach vertically integrating the operations on various levels (Adams et al. 2016a) yields excellent memory access characteristics per level plus in-between the level updates.

Our Helmholtz studies not only study higher-dimensional grids $(d \geq 4)$, they also discuss how the solution of multiple PDEs on one grid can be merged to yield higher arithmetic intensity. In this case, multiple PDEs are stored within one grid, and the arising stencils not only tackle the individual PDEs, they also couple them that yields additional flops. Our convection-diffusion studies compare the derived solvers to a setup where each vertex holds only one integer. This integer serves as local index to PETSc (Balay et al. 2016), i.e., we compare a matrix-free, monolithic solver with Peano to a solver where Peano yields solely the discretization, the user automaton 
realizes the assembly, and the actual equation system is administered and solved by a black-box solver. Our results suggest that Peano's matrix-free/integrated approach is superior to explicit assembly with PETSc if the grid changes frequently throughout the solve, i.e., if we realize for example full multigrid cycles that build up the grid setp by step or if we integrate a dynamic refinement criterion into the solve. If the grid is stationary, then an explicit assembly seems to be significantly faster.

Higher-order DG Discretizations. In the ExaHyPE project (Bader et al. 2015), we study Discontinuous Galerkin (DG) discretizations of first-order hyperbolic equations

$$
\frac{\partial}{\partial t} Q+\nabla \cdot F(Q)+\sum_{i} \mathcal{B}_{i} \frac{\partial Q}{\partial x_{i}}=S+\sum \delta
$$

on dynamically adaptive grids. They are subject to the explicit ADER-DG timestepping scheme. $Q$ here is defined cellwise as high-order $(p \in\{3,4, \ldots, 9\})$ polynomial over the cells of $\Omega_{h}$.

We make each Peano cell hold a pointer to an entry in Peano's heap. Each heap entry stores, per quantity in $Q,(p+1)^{d}$ weights of the Gauss Legendre shape functions. The spacetree acts as metadata structure. The actual compute data, i.e., $Q$, are held in the heap's hash map. ADER-DG is a predictor-corrector scheme: First, the automaton runs over $\Omega_{h, \ell}$ and computes a predicted solution to (4). This is done within enterCell. Second, we equip each vertex with $2^{d}$ pointers to the respective adjacent cells. This allows us to traverse the grid, i.e., all faces, and solve Riemann problems to the predicted solution there. The idea here is to plug into touchVertexFirstTime and to equip each face with one marker bit. The bit is set to zero by touchVertexLastTime. Once we enter touchVertexFirstTime, we analyse the $2 \cdot 2^{d-1}$ adjacent faces. For each face where our flagging bit is not set, we set it and evaluate the Riemann problem. Finally, we traverse the grid's cells and bring together the predicted solution with the Riemann solves. We exploit (1): it ensures that each vertex has been "touched" prior to enterCell. Consequently, all adjacent faces to a cell have been processed, too. It is one insight of the underlying project that this works with singletouch semantics, i.e., one grid sweep per time step.

Hanging vertices inherit the pointers from their parent vertices when the automaton triggers createHangingVertex. The parents of a vertex are all vertices on the next coarser level that are adjacent to a cell that in turn is a parent cell to a cell adjacent to the vertex of interest. With this definition, we can determine neighbouring cells of any hanging vertex and handle resolution transitions. ADER-DG quickly becomes unstable in the presence of shocks. For shocks, we apply a Finite Volume solver as limiter. If limiters are present, then our grid carries two solvers per cell.

Patch-based Finite Volume Solvers. In (Weinzierl et al. 2014), we study a standard Finite Volume solver for shallow water equations

$$
\frac{\partial}{\partial t}\left(\begin{array}{c}
h \\
h u \\
h v \\
b
\end{array}\right)+\frac{\partial}{\partial x}\left(\begin{array}{c}
h u \\
h u^{2}+0.5 g h^{2} \\
h u v \\
0
\end{array}\right)+\frac{\partial}{\partial y}\left(\begin{array}{c}
h v \\
h u v \\
h v^{2}+0.5 g h^{2} \\
0
\end{array}\right)+\left(\begin{array}{c}
0 \\
h g \cdot b_{x} \\
h g \cdot b_{y} \\
0
\end{array}\right)=0 .
$$

In the simplest variant (Figure 3), we apply a simple Rusanov flux. We store three unknown quantities per cell. In an original variant they are held within the spacetree stream. Per face, we have to hold the fluxes. As Peano does not natively provide face unknowns, we store the quantities from the positions $(x+h / 2, y)$ and $(x, y+h / 2)$ within the vertex at $(x, y) . h$ is the cell size. We map the staggered degree of freedom layout onto vertex and cell storage locations. Within each cell, we have, through the $2^{d}$ adjacent vertices, all data available to run the Finite Volume updates. 
Few codes solving equations like (5) use strongly adaptive meshes where single cells resolve particular properties. Adaptivity criteria here typically yield areas of refinement. We therefore next embed whole $n \times n$ patches into the individual cells, equip them with a halo layer of width one, and hold both the current and the previous solution within the patch. When the automaton triggers enterCell, we first fill the ghost layer and then trigger the Finite Volume scheme on the patch. This block-structured AMR also yields reasonable complex compute kernels per cell that can be vectorised. If patch-based processing first interpolates and then starts from the finest level, i.e., plugs into the DFS traversal's backtracking, then it mirrors the dataflow of classic local time stepping for hyperbolic equation systems with subcycling (cf. references in (Deiterding 2005; Dubey et al. 2016)).

There is an obvious trade-off between patch size $n$-the bigger $n$, the lower the spacetree's administration overhead and the higher the impact of classic stencil optimization techniques and vectorization-and degree of freedom per accuracy. In (Weinzierl et al. 2014) we apply a technique along the lines of (2) to our patches: Whenever we identify an assembly of $3 \times 3$ patches on a level $\ell$, we can replace this patch by one $3 n \times 3 n$ patch embedded into level $\ell-1$. The argument applies recursively. Our spacetree's multilevel nature thus allows us to use tiny patches with Finite Volume kernels that cannot optimise too aggressively. These patches are (temporarily, as long as refinement criteria allow us to do so) replaced by larger patches on coarser tree levels. For these, optimised kernels can be written. Once an adaptivity criterion requires us to refine, we first break up the fused patches, and then continue with classic block-structured AMR with tiny patches. This technique can be read as a predecessor to the present article's optimizations in Section 5.

Particle-grid Data Structures for Particle-in-Cell, the Discrete Element Method and SPH. Lagrangian methods and particle models often employ meshes as helper data structure to speed up operations such as collision detection or the evaluation of formula that are subject to a cut-off radius. In (Weinzierl et al. 2016), we derive a multiscale particle administration algorithm that scales. This algorithm is used for an SPH application in (Eckhardt et al. 2015) and as base of a discrete element method code in (Krestenitis et al. 2017) (Figure 2).

Both codes exploit the fact that each particle has a cut-off radius or a neighbourhood where we have to search for possible collisions, respectively. Both quantities determine a mesh size $h$ if we embed the objects into a grid. Given a certain nonstationary particle distribution, we create a spacetree that can accommodate the particles: it is refined down to the finest mesh size $h$, but particles are embedded into the level that suits their cut-off/search radius. To store them, each vertex is given a pointer to a dynamic range vector of particles. Each particle is stored to the vertex on the respective level next to its centre. The efficient on-the-fly administration of the respective particle lists is subject of discussion in (Weinzierl et al. 2016). On one level, we compare particles to each other within enterCell. Inter-grid operators in return compare particles living on various scales.

Limitations. While pointers from vertices to neighbouring cells allow the automaton per cell to access the neighbouring cells, Peano's strict element-wise automaton paradigm forbids codes to access neighours of neighbours or vertices of neighbouring cells. It might be possible to permit such freedom of access through additional helper structures along the lines of the vertex-to-cell pointers, but it then might be reasonable to assess completely alternative software solutions anyway. Therefore, we haven't investigated into this option yet.

Peano focuses on grid traversals through the whole tree. More anarchic temporal access patterns (process only cells of a certain resolution or carrying markers) as we find them in multiplicative multigrid for elliptic setups or local time stepping for hyperbolic equation system solvers can be realised by a marker approach where the grid traversal automaton process the whole grid but 
updates only particular grid entities. If the actual grid operations are expensive and can be deployed to dedicated threads, then we end up with a producer-consumer pattern: Peano efficiently traverses the tree and spawns tasks that are then evaluated on separate threads. If the spawned tasks are reasonably expensive, then such a pattern can scale, and Peano provides the required infrastructure for this.

The realisation of complex multilevel algorithms that require information propagation from coarse to fine levels and the other way round can straightforwardly be realised through multiple grid sweeps. Again, it is not clear however whether other grid organisation paradigms then would be more appropriate. As an alternative to multiple sweeps, one can investigate pipelining along the lines of (Charrier and Weinzierl 2017; Reps and Weinzierl 2017; Weinzierl 2013): Pipelining introduces helper variables per entity. These helper variables store additional information (about unknown updates, e.g.) such that grid traversals can be eliminated or reduced.

\section{BALANCING}

While grid balancing is not built into Peano's algorithms by construction, we offer a balancing extension. It can be compiled into any application without further need for user customization. It helps to ensure that the level of no two cells that are adjacent to each other, i.e., vertex-connected, differs by more than one.

The extension exploits Peano's or-based refinement convention (Weinzierl and Mehl 2011): Let a spacetree cell be refined if and only if at least one of its non-hanging adjacent vertices holds a refinement marker. The other way round, a marker on a vertex makes all of the vertex's adjacent cells refine. When the grid traversal automaton backtracks, it plugs into the storage of the persistent, i.e., non-hanging vertices (touchVertexLastTime). In our spacetree, a refined cell spans a $3 \times 3 \times \ldots$ patch on the next level. Any vertex on this next level consequently has a position $p \in\{0,1,2,3\}^{d}$ relative to the coarser refined cell. This enumeration is not unique-for vertices along the coarse grid faces, it depends on whether we look "from the right or the left"-but the nondeterminism does not affect the algorithm's outcome. If a fine grid vertex at $p$ holds a refinement marker and if any parent vertex of the vertex at $\hat{p}$ with $\hat{p}_{i}=\min \left(3\left(p_{i}-1\right), 3\right), i \in\{1,2, \ldots, d\}$ does not hold a refinement marker, then the grid is not balanced. We refine this parent vertex if it is not a hanging.

Peano's out-of-the-box 2:1 balancing eliminates too aggressive refinement incrementally. The above algorithm eliminates one level of ill-balancing between two adjacent cells. It thus can lead to rippling that propagates a refinement through the grid over several grid sweeps. For the aforementioned demonstrator applications, we rarely observed ill-balanced grids with more than two levels of resolution difference between adjacent cells, and we always have been able to accept that this ill-balancing is immediately resolved in the subsequent grid traversal. Any rippling integrates into the grid sweeps. If an algorithm requires immediate well-balancing after each grid modification, then users manually have to hold their computation once the premanufactured balancing identifies ill-balancing and have to insert iterative re-balancing grid sweeps. The cost per sweep corresponds to an AMR grid traversal without any computation. Alternatively, it might be reasonable to investigate into sophisticated grid balancing along the lines of (Sundar et al. 2008; Tu et al. 2005). This is however not offered out-of-the-box.

While the iterative, lazy elimination of ill-balancing so far has been sufficient for most of our applications, we found that elliptic solvers with non-homogeneous boundary conditions benefit from an additional constraint: Eliminate hanging nodes on the domain boundary. Whenever hanging vertices along the boundary are found, it is convenient for user codes to trigger refinement next to the boundary on the next coarser level. This way, they avoid that we underresolve the boundary and thus suffer from pollution effects from inaccurate boundary conditions. 


\section{ACKNOWLEDGMENTS}

Thanks are due to all the scientists and students who contributed to the software in terms of software fragments, applications, extensions, and critical remarks. Notably, thanks are due to HansJoachim Bungartz and his group at Technische Universität München, who provided the longestterm environment for the development of this code. The article is dedicated to Christoph Zenger, who kicked off and supervised the implementation of the first generation of Peano.

\section{REFERENCES}

M. Adams, P. Colella, D. T. Graves, J. N. Johnson, N. D. Keen, T. J. Ligocki, D. F. Martin, P. W. McCorquodale, D. Modiano, P. O. Schwartz, T. D. Sternberg, and B. Van Straalen. 2016b. Chombo-Software for Adaptive Solutions of Partial Differential Equations. Retrieved from https://commons.lbl.gov/display/chombo.

M. F. Adams, J. Brown, M. Knepley, and R. Samtaney. 2016a. Segmental refinement: A multigrid technique for data locality. SIAM 7. Sci. Comput. 38, 4 (2016), C426-C440.

V. Akcelik, J. Bielak, G. Biros, I. Epanomeritakis, A. Fernandez, O. Ghattas, E. J. Kim, J. Lopez, D. O’Hallaron, T. Tu, and J. Urbanic. 2003. High resolution forward and inverse earthquake modeling on terascale computers. In Proceedings of the 2003 ACM/IEEE Conference on Supercomputing (SC'03). ACM, New York, NY.

M. Bader. 2013. Space-Filling Curves-An Introduction with Applications in Scientific Computing. Texts in Computational Science and Engineering, Vol. 9. Springer-Verlag.

M. Bader, M. Dumbser, A.-A. Gabriel, H. Igel, L. Rezzolla, and T. Weinzierl. 2015. ExaHyPE-An Exascale Hyperbolic PDE solver Engine. Retrieved from http://www.exahype.org.

Satish Balay, Shrirang Abhyankar, et al. 2016. PETSc Web page. Retrieved from http://www.mcs.anl.gov/petsc.

W. Bangerth, C. Burstedde, T. Heister, and M. Kronbichler. 2011. Algorithms and data structures for massively parallel generic adaptive finite element codes. ACM Trans. Math. Softw. 38, 2 (2011).

W. Bangerth, D. Davydov, T. Heister, L. Heltai, G. Kanschat, M. Kronbichler, M. Maier, B. Turcksin, and D. Wells. 2016. The deal. II library, version 8.4. f. Numer. Math. 24, 3 (2016), 135-141.

W. Bangerth, R. Hartmann, and G. Kanschat. 2007. deal.II-A general-purpose object-oriented finite element library. ACM Trans. Math. Softw. 33, 4 (2007).

P. Bastian, M. Blatt, A. Dedner, C. Engwer, R. Klöfkorn, M. Ohlberger, and O. Sander. 2008a. A generic grid interface for parallel and adaptive scientific computing. Part I: Abstract framework. Computing 82, 2-3 (2008), 103-119.

P. Bastian, M. Blatt, A. Dedner, C. Engwer, R. Klöfkorn, M. Ohlberger, and O. Sander. 2008b. A generic grid interface for parallel and adaptive scientific computing. Part II: Implementation and tests in DUNE. Computing 82, 2-3 (2008), 121-138.

R. Bellman. 1961. Adaptive Control Processes: A Guided Tour. Princeton University Press.

B. K. Bergen and F. Hülsemann. 2004. Hierarchical hybrid grids: Data structures and core algorithms for multigrid. Numer. Lin. Algebr. Appl. 11, 2-3 (2004), 279-291.

A. Brandt. 1973. Multi-level adaptive technique (MLAT) for fast numerical solution to boundary value problems. In Proceedings of the 3rd International Conference on Numerical Methods in Fluid Mechanics, Lecture Notes in Physics. 82-89.

A. Brandt. 1977. Multi-level adaptive solutions to boundary-value problems. Math. Comp. 31, 138 (1977), 333-390.

H.-J. Bungartz, W. Eckhardt, T. Weinzierl, and Ch. Zenger. 2010a. A precompiler to reduce the memory footprint of multiscale PDE solvers in C++. Fut. Gen. Comput. Syst. 26, 1 (2010), 175-182.

H.-J. Bungartz, B. Gatzhammer, M. Lieb, M. Mehl, and T. Neckel. 2011. Towards multi-phase flow simulations in the PDE framework Peano. Comput. Mech. 48, 3 (2011), 365-376.

H.-J. Bungartz, M. Mehl, T. Neckel, and T. Weinzierl. 2010b. The PDE framework Peano applied to fluid dynamics: An efficient implementation of a parallel multiscale fluid dynamics solver on octree-like adaptive Cartesian grids. Comput. Mech. 46, 1 (2010), 103-114.

H.-J. Bungartz, M. Mehl, and T. Weinzierl. 2006. A parallel adaptive Cartesian PDE solver using space-filling curves. In Proceedings of the International European Conference on Parallel and Distributed Computing (Euro-Par'06), W. E. Nagel, W. V. Walter, and W. Lehner (Eds.), Lecture Notes in Computer Science, Vol. 4128. Springer-Verlag, Berlin, 1064-1074.

H.-J. Bungartz, M. Mehl, T. Weinzierl, and W. Eckhardt. 2008. DaStGen - A data structure generator for parallel C++ HPC software. In Proceedings of the International Conferences on Conceptual Structures: Advancing Science through Computation, Part III (ICCS'08), M. Bubak, G. D. van Albada, P. M. A. Sloot, and J. Dongarra (Eds.), Lecure Notes in Computer Science, Vol. 5103. Springer-Verlag, Berlin, 213-222.

C. Burstedde, J. Holke, and T. Isaac. 2017. Bounds on the Number of Discontinuities of Morton-type Space-filling Curves. Technical Report. arXiv:1505.05055. 
C. Burstedde, L. C. Wilcox, and O. Ghattas. 2011. p4est: Scalable algorithms for parallel adaptive mesh refinement on forests of octrees. SIAM 7. Sci. Comput. 33, 3 (2011), 1103-1133.

D. E. Charrier and T. Weinzierl. 2017. An experience report on (auto-)tuning of mesh-based PDE solvers on shared memory systems. In Proceedings of the International Conference on Parallel Processing and Applied Mathematics (PPAM'17), R. Wyrzykowski, J. Dongarra, E. Deelman, and K. Karczewski (Eds.), Lecture Notes in Computer Science, Vol. 10778. 3-13.

D. E. Charrier and T. Weinzierl. 2018. Stop talking to me - A communication-avoiding ADER-DG realisation. (2018). arXiv:1801.08682.

J. Davison de St. Germain, J. McCorquodale, S. G. Parker, and C. R. Johnson. 2000. Uintah: A massively parallel problem solving environment. In Proceedings of the 9th International Symposium on High-Performance Distributed Computing (2000), 33-41.

R. Deiterding. 2005. Detonation structure simulation with AMROC. In High Performance Computing and Communications, L. T. Yang, O. F. Rana, B. Di Martino, and J. Dongarra (Eds.), Lecture Notes in Computer Science, Vol. 3726. 916-927.

J. Dreher and R. Grauer. 2005. Racoon: A parallel mesh-adaptive framework for hyperbolic conservation laws. Parallel Comput. 31, 8-9 (2005), 913-932.

A. Dubey, A. S. Almgren, J. B. Bell, M. Berzins, S. R. Brandt, G. Bryan, P. Colella, D. T. Graves, M. Lijewski, F. Löffler, B. O'Shea, E. Schnetter, B. van Straalen, and K. Weide. 2016. A survey of high level frameworks in block-structured adaptive mesh refinement packages. F. Parallel Distrib. Comput. 74, 12 (2016), 3217-3227.

W. Eckhardt, R. Glas, D. Korzh, S. Wallner, and T. Weinzierl. 2015. On-the-fly memory compression for multibody algorithms. In Advances in Parallel Computing, Vol. 27. 421-430.

W. Eckhardt and T. Weinzierl. 2010. A blocking strategy on multicore architectures for dynamically adaptive PDE solvers. In Proceedings of the International Conference on Parallel Processing and Applied Mathematics (PPAM'09), R. Wyrzykowski, J. Dongarra, K. Karczewski, and J. Wasniewski (Eds.), Lecture Notes in Computer Science, Vol. 6068. Springer-Verlag, 567-575.

C. Feichtinger, S. Donath, H. Köstler, J. Götz, and U. Rüde. 2011. WaLBerla: HPC software design for computational engineering simulations. F. Comput. Sci. 2, 2 (2011), 105-112.

G. Brito Gadeschi, L. Schneiders, M. Meinke, and W. Schröder. 2015. A numerical method for multiphyics simulations based on hierarchical Cartesian grids. f. Fluid Sci. Technol. 10, 1 (2015), 1-12.

E. Gamma, R. Helm, R. E. Johnson, and J. Vlissides. 1994. Design Patterns-Elements of Reusable Object-Oriented Software (1st ed.). Addison-Wesley Longman.

C. Gotsman and M. Lindenbaum. 1996. On the metric properties of discrete space-filling curves. IEEE Trans. Image Process. 5, 5 (1996), 794-797.

M. Grandin. 2015. Data structures and algorithms for high-dimensional structured adaptive mesh refinement. Adv. Eng. Softw. 82, C (2015), 75-86.

M. Grandin and S. Holmgren. 2014. Parallel Data Structures and Algorithms for High-Dimensional Structured Adaptive Mesh Refinement. Technical Report 20. Uppsala Universitet.

M. Griebel and G. Zumbusch. 1998. Hash-storage techniques for adaptive multilevel solvers and their domain decomposition parallelization. In Proceedings of the Conference on Domain Decomposition Methods (DD10), Contemporary Mathematics, Vol. 218. 279-286.

M. Griebel and G. Zumbusch. 1999. Parallel multigrid in an adaptive PDE solver based on hashing and space-filling curves. Parallel Comput. 25, 7 (Jul. 1999), 827-843.

H. Haverkort. 2016. How many three-dimensional Hilbert curves are there? arXiv:1610.00155 (2016).

J. Hungershöfer and J. M. Wierum. 2002. On the quality of partitions based on space-filling curves. In Proceedings of the International Conference on Computational Science 2002, Lecture Notes in Computer Science, Vol. 2331. 31-45.

T. Isaac, C. Burstedde, and O. Ghattas. 2012. Low-cost parallel algorithms for 2:1 octree balance. In Proceedings of the IEEE 26th International Parallel and Distributed Processing Symposium. 426-437.

J.-H. Jeong, N. Goldenfeld, and J. A. Dantzig. 2001. Phase field model for three-dimensional dendritic growth with fluid flow. Phys. Rev. E 64, 4 (Sep. 2001), 041602.

A. M. Khokhlov. 1998. Fully threaded tree algorithms for adaptive refinement fluid dynamics simulations. f. Comput. Phys. 143, 2 (1998), 519-543.

D. E. Knuth. 1990. The genesis of attribute grammars. In WAGA: Proceedings of the International Conference on Attribute Grammars and Their Applications, P. Deransart and M. Jourdan (Eds.). Springer-Verlag, 1-12.

V. Kolobov and R. Arslanbekov. 2016. Electrostatic PIC with adaptive Cartesian mesh. J. Phys. Conf. Ser. 719, 1 (2016), 012020 .

M. Kowarschik and C. Weiß. 2003. An overview of cache optimization techniques and cache-aware numerical algorithms. In Proceedings of the Algorithms for Memory Hierarchies 2002, U. Meyer, P. Sanders, and J. F. Sibeyn (Eds.). Springer-Verlag, 213-232. 
K. Krestenitis, T. Weinzierl, and T. Koziara. 2017. Fast DEM collision checks on multicore nodes. In Proceedings of the International Conference on Parallel Processing and Applied Mathematics (PPAM'17), R. Wyrzykowski et al. (Eds.), Lecture Notes in Computer Science, Vol. 10777. 123-132.

I. Lashuk, A. Chandramowlishwaran, H. Langston, T.-A. Nguyen, R. Sampath, A. Shringarpure, R. Vuduc, L. Ying, D. Zorin, and G. Biros. 2012. A massively parallel adaptive fast multipole method on heterogeneous architectures. Commun. ACM 55, 5 (2012), 101-109.

J. D. McCalpin. 1995. Memory bandwidth and machine balance in current high performance computers. In Proceedings of the IEEE Computer Society Technical Committee on Computer Architecture (TCCA'95) Newsletter (Dec. 1995), 19-25.

S. F. McCormick. 1989. Multilevel Adaptive Methods for Partial Differential Equations. SIAM.

M. Mehl, T. Weinzierl, and C. Zenger. 2006. A cache-oblivious self-adaptive full multigrid method. Numer. Lin. Algebr. Appl. 13, 2-3 (2006), 275-291.

O. Meister, K. Rahnema, and M. Bader. 2012. A software concept for cache-efficient simulation on dynamically adaptive structured triangular grids. In Proceedings of the International Conference on Parallel Computing (ParCo'12), K. De Boschhere, E. H. D’Hollander, G. R. Joubert, D. Padua, and F. Peters (Eds.), Advances in Parallel Computing, Vol. 22. IOS Press, 251-260.

P. Neumann. 2013. Hybrid Multiscale Simulation Approaches For Micro- and Nanoflows. Verlag Dr. Hut, München.

A. Rahimian, I. Lashuk, S. Veerapaneni, A. Chandramowlishwaran, D. Malhotra, L. Moon, R. Sampath, A. Shringarpure, J. Vetter, R. Vuduc, D. Zorin, and G. Biros. 2010. Petascale direct numerical simulation of blood flow on 200K cores and heterogeneous architectures. In Proceedings of the International Conference for High Performance Computing, Networking, Storage, and Analysis (SC'10). 1-11.

S. Reiter, A. Vogel, I. Heppner, M. Rupp, and G. Wittum. 2013. A massively parallel geometric multigrid solver on hierarchically distributed grids. Comput. Vis. Sci. 16, 4 (2013), 151-164.

B. Reps and T. Weinzierl. 2017. A complex additive geometric multigrid solver for the Helmholtz equations on spacetrees. ACM Trans. Math. Softw. 44, 1 (2017), 2:1-2:36. DOI : http://doi.acm.org/10.1145/3054946

R. N. Robey, D. Nicholaeff, and R. B. Robey. 2013. Hash-based algorithms for discretized data. SIAM 7. Sci. Comput. 35, 4 (2013).

R. S. Sampath, S. S. Adavani, H. Sundar, I. Lashuk, and G. Biros. 2008. Dendro: Parallel algorithms for multigrid and AMR methods on 2:1 balanced octrees. In Proceedings of the 2008 ACM/IEEE Conference on Supercomputing. IEEE Press, Article 18,12 pages.

F. Schornbaum and U. Rüde. 2018. Software and high-performance computing extreme-scale block-structured adaptive mesh refinement. SIAM 7. Sci. Comput. 40, 3 (2018), C358-C387.

M. Schreiber, T. Weinzierl, and H.-J. Bungartz. 2013b. Cluster optimization and parallelization of simulations with dynamically adaptive grids. In Proceedings of the International European Conference on Parallel and Distributed Computing (Euro-Par'13), F. Wolf, B. Mohr, and D. an Mey (Eds.), Vol. 8097. Springer-Verlag, Berlin, 484-496.

M. Schreiber, T. Weinzierl, and H.-J. Bungartz. 2013a. SFC-based communication metadata encoding for adaptive mesh refinement. In Advances in Parallel Comput., M. Bader (Ed.), Vol. 25. 233-242.

J. Skilling. 2004. Programming the Hilbert curve. AIP Conf. Proc. 707, 381 (2004).

H. Sundar, G. Biros, C. Burstedde, J. Rudi, O. Ghattas, and G. Stadler. 2012. Parallel geometric-algebraic multigrid on unstructured forests of octrees. In Proceedings of the International Conference on High Performance Computing, Networking, Storage and Analysis (SC'12). IEEE Computer Society Press, Los Alamitos, CA, Article 43, 11 pages.

H. Sundar and O. Ghattas. 2015. A Nested Partitioning Algorithm for Adaptive Meshes on Heterogeneous Clusters. Vol. 2015June. Association for Computing Machinery, 319-328.

H. Sundar, R. S. Sampath, and G. Biros. 2008. Bottom-up construction and 2:1 balance refinement of linear octrees in parallel. SIAM 7. Sci. Comput. 30, 5 (Aug. 2008), 2675-2708.

R. E. Sweet. 1985. The mesa programming environment. SIGPLAN Not. 20,7 (1985), 216-229.

J. Teunissen and U. Ebert. 2017. Afivo: A framework for quadtree/octree AMR with shared-memory parallelization and geometric multigrid methods. Comput. Phys. Commun. 233 (2017), 156-166.

T. Tu, D. R. O'Hallaron, and O. Ghattas. 2005. Scalable parallel octree meshing for terascale applications. In Proceedings of the 2005 ACM/IEEE Conference on Supercomputing (SC'05). IEEE Computer Society, Los Alamitos, CA, 4-. DOI : https:// doi.org/10.1109/SC.2005.61

R. Tumblin, P. Ahrens, S. Hartse, and R. W. Robey. 2015. Parallel compact hash algorithms for computational meshes. SIAM f. Sci. Comput. 37, 1 (2015), 31-53.

B. Turcksin, M. Kronbichler, and W. Bangerth. 2016. WorkStream-A design pattern for multicore-enabled finite element computations. ACM Trans. Math. Softw. 43, 1 (2016), 2:1-2:29.

J. Weinbub, K. Rupp, and S. Selberherr. 2014. Highly flexible and reusable finite element simulations with ViennaX. F. Comput. Appl. Math. 270 (2014), 484-495.

M. Weinzierl. 2013. Hybrid Geometric-Algebraic Matrix-Free Multigrid on Spacetrees. Dissertation. Fakultät für Informatik, Technische Universität München, München. Retrieved from http://mediatum.ub.tum.de/doc/1138173/1138173.pdf. 
M. Weinzierl and T. Weinzierl. 2018. Algebraic-geometric matrix-free multigrid on dynamically adaptive Cartesian meshes. ACM Trans. Math. Softw. 44, 3 (2018), 32:1-32:44.

T. Weinzierl. 2009. A Framework for Parallel PDE Solvers on Multiscale Adaptive Cartesian Grids. Verlag Dr. Hut.

T. Weinzierl et al. 2015. Peano-A Framework for PDE Solvers on Spacetree Grids. Retrieved from http://www. peano-framework.org.

T. Weinzierl, M. Bader, K. Unterweger, and R. Wittmann. 2014. Block fusion on dynamically adaptive spacetree grids for shallow water waves. Parallel Process. Lett. 24, 1441006 (2014).

T. Weinzierl and M. Mehl. 2011. Peano-A traversal and storage scheme for octree-like adaptive cartesian multiscale grids. SIAM 7. Sci. Comput. 33, 5 (2011), 2732-2760.

T. Weinzierl, B. Verleye, P. Henri, and D. Roose. 2016. Two particle-in-grid realisations on spacetrees. Parallel Comput. 52 (2016), 42-64.

Received August 2017; revised December 2018; accepted December 2018 